\title{
Integrated Supply Chain Management of Vegetables in Jhansi Division of Uttar Pradesh
}

Ram Kumar Jha* and Anshul Kumar Jain $†$

\section{Abstract}

The study has been made on backward Jhansi division of Uttar Pradesh. Two stage stratified random sampling method has been adopted to collect the information from vegetables growers. Total 114 vegetables growers categorized marginal, small and large farmers, 22 middlemen and 20 retailers have been chosen to complete the present study. Objectives of the study are i) to examine the trends and percent growth rates for the area, production and productivity of different vegetable crops; ii) to estimate the trend for arrival and market prices of different vegetable crops; iii) to calculate the yearly arrival index for vegetables arrival in selected mandies, and iv) to judge integrated supply chain management of vegetables marketing in selected region.

Middlemen purchased vegetables at six percent commission on per quintal basis from producers and sold to retailer by charging three percent mandishulk on per quintal basis. Middlemen also provide space to keep their produce and stay at night for farmers in mandi.

*Policy Analyst, CUTS International, D-217, Bhaskar Marg, Bani Park, Jaipur, Rajasthan and Visiting Professor, CCDR, Lalitpur (UP); ram_econ@yahoo.co.in

†Research Scholar, Department of Management, JJTU, Jhunjhunu (Rajasthan) at Centre for Communication \& Development Research, Lalitpur (Uttar Pradesh) as research centre;anshul.kcc@gmail.com 
The study finds that Lalitpur and Jhansi districts have potential to increase the productivity of tomato through better governmental support. Under the marketing chain management the channel II was found most favored channel in the study area, as maximum (about 90\%) quantity of produce was sold through this channel by small and large farmers.

Keywords: Arrivals, Market prices, Retention, Marketing chain management, Vegetables

\section{Introduction}

Horticultural products play a major role in modern society and economy. The constant growth in vegetables productivity is important for stability of the Indian economy with improving the productivity of land, generating employment throughout the year, improving economic conditions of the farmers and entrepreneurs, enhancing exports, and providing nutritional security to the people. Vegetables are part of everyday meals, including special high-value diets for infants and the elderly.

Uttar Pradesh achieved only three percent growth against the planned four percent for agriculture sector in eleventh five years plan. Therefore, in twelfth five years plan, to achieve the multidimensional potential of agricultural development in the state, government has made necessary changes in the present Agriculture Policy 2013 keeping in view the future challenges of the state.

It is mentioned in National Horticulture Board (NHB), Report 2013 that in vegetables production, India is next to China with an annual production of 87.53 million tons from 5.86 million hectares (Mha) having a share of 14.4 per cent to the world production. Per capita consumption has also increased from 95 gram to 175 gram per day. Potato is the most widely grown vegetable crop in the country with a share of 25.7 per cent. The area under potato cultivation is 1992.2 thousand ha with total production of 45343.6 thousand metric ton (MT). Uttar Pradesh is the leading potato growing state in the country with a production of 13576.6 thousand MT followed by West Bengal and Bihar in 2013. Tomato occupies 
second position amongst the vegetable crops in terms of production. The total production of tomato in the country in 201213 is 18226.6 thousand MT from an area of 879.6 Andhra Pradesh is the largest grower of tomato with a production of 5218.10 thousand MT. The other main tomato growing states are Bihar, Karnataka, Maharashtra and Orissa. Brinjal occupies the third position amongst vegetable crops. The production of brinjal in the year 2012-13 was 13443.6 thousand MT from an area 722.1 Tha. West Bengal is the largest producer of brinjal followed by Maharashtra and Bihar. The other main states growing brinjal are Karnataka, Maharashtra, Gujarat, Andhra Pradesh, Assam and Madhya Pradesh. Cauliflower occupies the sixth position amongst vegetable crops. The production of cauliflower in the year 2012-13 was 7887 thousand MT from an area 402 Tha. West Bengal is the largest producer of cauliflower followed by Bihar, Madhya Pradesh and Maharashtra. The other main states growing cauliflower are Karnataka, Maharashtra, Gujarat, Andhra Pradesh, and Assam. Okra occupies the good position amongst vegetable crops. The production of okra in the year 2012-13 was 6350 thousand MT from an area 231 Tha. Andhra Pradesh is the largest producer of Okra followed by West Bengal, Bihar and Maharashtra. The other main states growing of okra are Karnataka, Maharashtra, Gujarat, Assam and Madhya Pradesh. Onion occupies the third position amongst vegetable crops. The production of onion in the year 2012-13 was 16813 thousand MT from an area 1052 Tha. Maharashtra is the largest producer of onion followed by Madhya Pradesh and Karnataka. Bihar, Gujarat, Haryana and Rajasthan are the other onion growing states.

Farmers are shifting from staple crops to high-value cash crops farming such as fruits, and vegetables. This adaptation from subsistence systems to commercial agriculture poses new challenges for improving and maintaining productivity and quality to secure food security. Supply chain of horticulture is the big issue being a perishable commodity. Potato, onion can be stored for few months but other than these produce need markets to sale. Proper supply chain and suitable price attract farmers to sell their produces. Supply chain management plays an integral role in keeping business costs at a minimum and profitability as high as possible. Farmers producing agricultural produce are scattered in 
remote villages while consumers are in semi-urban and urban areas. There are different marketing channels through which this produce reaches the consumer for its final use and consumption.

Agricultural produce market committee (APMC) Act has the clause to strengthen public-private approach in agricultural marketing. But the UP government did not adopt this act due to external pressure of middlemen (Amar Ujala, 2014). Kasturi (2014) pointed that the traders being the main beneficiaries of the raised prices of vegetables over an extended period this year. The trigger for rising prices can be varied - a lower harvest, an unexpected rise in demand, damage to standing crops, or even delayed sowing. Most farmers - lacking the ability to store produce - would not have gained as prices remained low in the harvest period. According to the World Bank (2007), the food supply chain linking food production with food consumption and human nutrition can be considered in terms of five pathways, namely, (1) subsistenceoriented production for the household's own consumption, (2) Income-oriented production for sale in markets, (3) reduction in real food prices associated with increased agricultural production, (4) empowerment of women as agents instrumental to household food security and health outcomes, and (5) indirect relationship between increasing agricultural productivity and nutrition outcomes through the agriculture sector's contribution to national income and macroeconomic growth.

Minister of State for Agriculture Tariq Anwar was speaking that the vegetable supply chain is crying out for reform. The government is well aware of what ails the supply chain, as the quote below shows. If farmers get paid Rs 10 per $\mathrm{kg}$, while the consumers pay anywhere between Rs 80-100 per $\mathrm{kg}$ of onion, there is obviously something seriously wrong in the way onion market operates ... we know that there are governance issues related to licensing of wholesalers, issues related to holding capacity of farmers, issues related to market prices information and a number of issues related to the whole supply chain which creates the kind of pricing contradiction (Anwar, 2013).

Though vegetables retail has been considered as a very low-margin business but the market potential has attracted Indian business 4 
houses and corporate, driving the forays through different models like single-format, multi format or integrated urban-rural models (Sengupta, 2008). Therefore, an attempt has been made in this paper i) to examine the trends and percent growth rates for the area, production and productivity of different vegetable crops; ii) to estimate the trend for arrival and market prices of different vegetable crops; iii) to calculate the yearly arrival index for vegetables arrival in selected mandies, and iv) to judge integrated supply chain management of vegetables marketing in selected region.

\section{Methodology}

The study has been made on backward division of Uttar Pradesh named Jhansi division. Division comprises three districts: Jhansi, Lalitpur and Jalaun on the basis of secondary and primary information.

\subsection{Secondary Data}

The secondary information is collected for area, production, productivity, arrival and market prices of tomato, brinjal, potato, onion, okra, bottle gourd, torai and cauliflower in Jhansi, Lalitpur, Jalaun districts and Jhansi division. The data has been compiled from various government department of Uttar Pradesh viz. Department of Horticulture and Food Processing, Uttar Pradesh (Statistics Department), Directorate of Agriculture and Marketing, Uttar Pradesh, District Agriculture Offices of Jhansi, Lalitpur and Jalaun districts. The data were available from 2005 to 2012 for the selected study area.

\subsection{Percent Growth Rates (PGR)}

To find out the percent growth rates and trend in Production, Area, and Productivity of selected vegetables in selected areas the following formula has been adopted.

$$
\text { Percent Growth Rates }(\mathrm{PGR})=\frac{Y 2-Y 1}{Y 1} \times 100
$$

Where,

$\mathrm{Y}_{1}=$ First year's area /production/ productivity of vegetables crop 
$\mathrm{Y}_{2}=$ Last year's area/ production/ productivity of vegetables crop

\subsection{Yearly Arrival Index}

Yearly Arrival Index has been calculated with the help of following formula.

$$
\text { Yearly Arrival Index }=\frac{\text { Yearly Arrival }}{\text { Average Arrival }} \times 100
$$

\subsection{Primary Data}

The primary information has been collected from Lalitpur district of Jhansi division. There are six blocks in Lalitpur district of Uttar Pradesh (UP) viz. Bar, Birdha, Jakhoura, Madavra, Mahroni, and Talbehat. Two stage stratified random sampling method has been adopted to collect the information from vegetables growers. At first stage, three blocks i.e., Mahroni, Birdha and Jakhoura were selected through random sampling method. Pre-tested questionnaire has been used to collect the information from vegetables growers at second stage and total 114 vegetables growers categorized marginal (up to 2.5 acre land), small (> 2.5 acre land to 5 acre land) and Large (> 5 acre land) farmers, 22 middlemen (aadatiya) and 20 retailers have chosen to complete the present study. The reference year was 2012-13.

\subsection{Limitations of the Study}

a) Unavailability of secondary information in the government offices because the record were not maintained properly.

b) It was very difficult to get information from the farmers at a first visit because of their illiteracy and hesitation.

\section{Results and Discussion}

\subsection{Vegetable Economy of Selected Regions}

This section gives a brief account of vegetables economy of selected regions. This is examined in terms of the production and productivity of major vegetables at the district and divisional level. This is followed by a brief account of the market arrival and market prices along with arrival index of products in different selected mandies. 
Table 1 outlines area, production and productivity of tomato, brinjal, potato, onion, okra, bottle gourd, torai and cauliflower in Jhansi, Lalitpur, Jalaun districts and Jhansi division. The maximum cultivated area for potato was in all three selected districts followed by onion. Much variation was not found in productivity of brinjal, potato, onion, okra, bottle gourd, torai and cauliflower. In Lalitpur district tomato production was highest in 2009-10 it was 2821 metric ton (MT). It was due to rise in area. The productivity throughout the selected time period was not fluctuated much more. Among the three districts maximum area covered by Jalaun district for tomato cultivation. It is seen that highest productivity for onion was in 2011-12 in Lalitpur district i.e., 22 metric ton/hectare. It was also found that the tomato productivity was also highest in Jalaun district. Therefore, Lalitpur and Jhansi districts have potential to increase the productivity of tomato through better governmental support.

Table 2 deals with yearly percent growth rates (PGR) of area, production and productivity of tomato, brinjal, potato, onion, okra, bottle gourd, torai and cauliflower in Jhansi, Lalitpur, Jalaun districts and Jhansi division. It informed regarding percentage change in present year compare to previous year. PGR shows that the selected area had much variation in cultivated area and production for selected vegetables.

Table 3 shows arrival, average market price and arrival indices for selected vegetables in selected areas. The total arrival indicated presence of vegetables in the different vegetable mandies and arrival index presented vegetables consistent presence in the mandies i.e., higher the index higher amount of quantity arrived in the mandies.

\subsection{Supply Chain Management}

The existing supply chain management channels of vegetables marketing by which the sampled farmers disposed of their surplus produce were found to be Middlemen and retailers. Some consumer's purchase directly from the producers at the village levels thereby saving on it and thus save marketing expenses. Sometimes, the producers directly take their vegetables produce to sale to the consumers. They pay Rs. $10-15$ per quintal more than the 
market price but in turn save all other marketing expenses. But at the time when supply increases consumers pay a significantly lesser amount to the producers for their produce. The supply chain management channels are present in below chart 1.The performance of these channels is presented below:

Channel I: Producer - Consumer (P-C): It was observed in the present study that producers directly sold their vegetables to consumers in rural, semi-urban and urban areas. There was no role of middlemen / intermediaries (aadatiya) in this channel. In this channel, the producer enjoyed cent percent share in the price paid by consumer (table 4 ).

Channel II: Producer - Middlemen - Retailer - Consumer (P-M$\mathrm{R}-\mathrm{C})$ : The outcome of the survey is that this channel is very common for all categories of farmers. In this channel producers sold their produce to Middlemen (aadatiya) and Middlemen sold to retailers and retailers sold to consumers. The end price of the produce is quite high and the producer's share in the price paid by the consumer has been observed quite low (table 4). This implies that the profit share of producer reduced while the consumers got the vegetables at higher price.

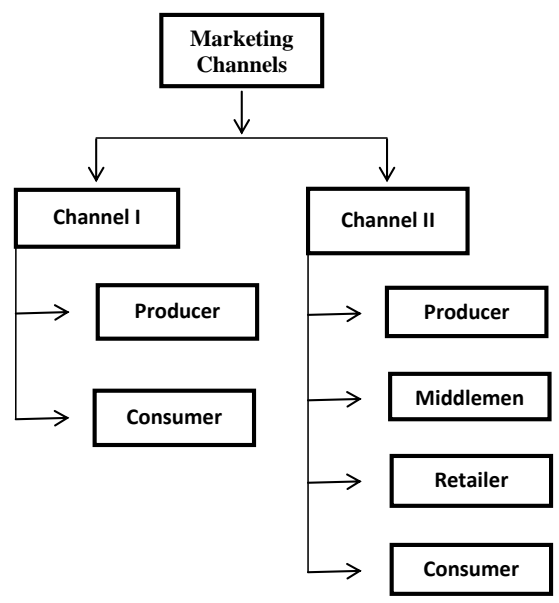

Chart 1: Supply Chain Management of Vegetables in Lalitpur District 


\subsection{Grading, Transportation and Storing}

In the selected study area vegetables growers were not grading their produce. They picked vegetables from their field and make bora bandi to carry in mandi. On the other hand the middlemen and retailers were grading the purchased vegetables for earning maximum profit.

Vegetables growers, middlemen and retailers used tractors, buses and taxis to carry vegetables from village to Lalitpur vegetable mandi. The vegetables growers of Mahroni block were using buses from block to Lalitpur bus stand and after that they used haththela and rickshaw from bus stand to mandi. From Birdha block, vegetables growers were coming by taxies and from Jakhoura block vegetables growers were bringing their produce through small loading truck (chhotahathi) and taxies. For direct selling (Producer to Consumer), vegetables growers and retailers used cycle and haththela to sell vegetables at towns and villages.

Vegetables growers did not store vegetables in home and warehouse. They were taking some amount for their home consumption. Even though, some middlemen were storing potato and onion at their shop for selling out in city at maximum price.

\subsection{Production, Retention and Marketed Surplus}

Table 4 outlines the total production, retention and marketed surplus of selected vegetables in selected areas. Marginal, small and large farmers sold more than 90 percent of their vegetables production into the market. The reason behind is this vegetables are considered as crops and help to fulfill farmer's daily needs and secondly vegetables are perishable and consumed within two to three days except potato and onion because these can be stored for few months. In all selected study areas retention rate was higher (60.5 percent) for Bottle Gourd in Birdha block for marginal farmers followed by large farmers (20.4) in Mahroni block. This shows that farmers were consuming some percentage of their vegetable produce even for some of the vegetables retention was zero means these were sold in to the market. Under the supply chain management the channel II was found most favored channel in the study area as maximum (about $90 \%$ ) quantity of produce was sold 
with this channel by small and large farmers. Channel I was mostly liked by marginal farmers.

\subsection{Role of Intermediaries in Vegetables Supply Chain Management}

Middlemen / wholesalers / commission agents played important role in vegetables marketing. They purchased vegetables at 6 percent commission on per quintal basis from producers and sold to retailer by charging 3 percent mandi shulk on per quintal basis. Thereafter, middlemen / commission agents were responsible for depositing mundi shulk in municipality.

Table 4 depicts how much quantity of vegetables produce was sold to middlemen from total marketed surplus of both the channels. This confers middlemen's role in vegetables supply chain in the study area. In Jakhoura block, all the selected farm size categories and in Birdha block small and large farm size categories sold their cent percent vegetables produce to middlemen. Marginal farmers sold cent percent produce of Onion and Okra in Mahroni block and Torai in Birdha block to middlemen. Middlemen also provide space to keep their produce and stay at night for farmers in mandi. At some occasions middlemen also provide credits to farmers for fulfilling their agricultural and family daily needs.

\section{Conclusions}

On the basis of above analysis it is concluded that Lalitpur district was on top in tomato production in 2009-10 with 2821 metric ton (MT). It was due to rise in area. The productivity throughout the selected period did not fluctuate much. Among the three districts maximum area covered by Jalaun district for tomato cultivation. Lalitpur and Jhansi districts have potential to increase the productivity of tomato by better governmental support. Percent Growth Rates (PGR) informed regarding percentage change in present year compare to previous year. PGR shows that the selected cultivated area under selected vegetables and production have been showing much variation for selected vegetables.

In the selected study area vegetables growers were not grading their produce. They picked vegetables from field and make bora bandi to carry in mandi. On the other hand the middlemen and 10 
retailers were grading the purchased vegetables for earning maximum profit. Preferred transportation modes were tractors, buses and taxies. But for retail selling cycle and haththela were mostly used. Vegetables growers did not store vegetables in home and warehouse.

The total arrival indicated presence of vegetables in the different vegetable mandies and yearly arrival index shows vegetables consistent presence in the mandies i.e., higher the yearly arrival index higher amount of quantity arrived in the mandies. In all selected study areas retention rate was higher (60.5 percent) for Bottle Gourd in Birdha block for marginal farmers followed by large farmers (20.4) in Mahroni block. This shows that farmers were consuming some percentage of their vegetable produce even for some of the vegetables retention was zero means these were sold in to the market. Under the supply chain management the channel II was found most favored channel in the study area as maximum (about 90\%) quantity of produce was sold with this channel by small and large farmers. Though channel I mostly liked by marginal farmers.

Middlemen purchased vegetables at 6 percent commission on per quintal basis from producers and sold to retailer by charging 3 percent mandishulk on per quintal basis. In Jakhoura block all the selected farm size categories and in Birdha block small and large farm size categories were sold their cent percent vegetables produce to middlemen. Middlemen also provide space to keep their produce and stay at night for farmers in mandi. At some occasions middlemen also provide credits to farmers for fulfilling their agricultural and family daily needs.

Therefore, value chain promotion is an effective way of fostering rural-urban linkages. Shifting towards cash crops cultivation requires proper marketing facilities. Government should re-think on issues of better price and marketing facilities for vegetables products producers. 


\section{References}

Amar Ujala (2014). Uttar Pradesh farmers shifting to high value cash crops, Hindi daily news paper, New Delhi, Sunday, August 10. Retrieved from http://www.amarujala.com/news/samachar/business/upfarmers-shifting-to-high-value-cash-crops-hindi-news / accessed on August 10, 2014.

Anwar, T. (2013), Governance deficit pushing onion prices upwards: MoS for agriculture Tariq Anwar, Economic Times, 8 November 2013.

Kasturi, K (2014), Have farmers benefitted from high vegetable prices in 2013?,Economic \& Political Weekly, XLIX(5), pp 14-17

NHB Report (2013), National Horticulture Board, Government of India. Retrieved from http://nhb.gov.in/

Sengupta, A. (2008), Emergence of modern Indian retail: Anhistorical perspective, International Journal of Retail $\mathcal{E}$ Distribution Management, 36(9), pp 689-700.

World Bank (2007), From Agriculture to Nutrition: Pathways, Synergies and Outcomes, Report No 40196-GLB (Washington DC: The International Bank for Reconstruction and Development / The World Bank). 
Table: 1Area, Production and Productivity of Selected Vegetables (2005 -06 to 2011-12)

\begin{tabular}{|c|c|c|c|c|c|c|c|c|c|c|c|c|c|}
\hline \multirow[b]{2}{*}{$\begin{array}{l}\frac{0}{0} \\
\frac{\pi}{0} \\
\stackrel{0}{0} \\
\stackrel{0}{D}\end{array}$} & \multirow[b]{2}{*}{ 䒕 } & \multicolumn{3}{|c|}{ Lalitpur } & \multicolumn{3}{|c|}{ Jhansi } & \multicolumn{3}{|c|}{ Jalaun } & \multicolumn{3}{|c|}{ Jhansi Division } \\
\hline & & 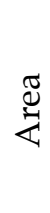 & 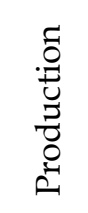 & 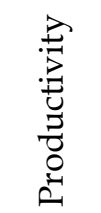 & 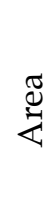 & 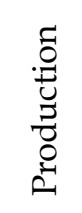 & 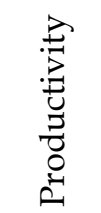 & 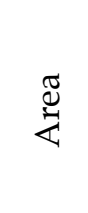 & 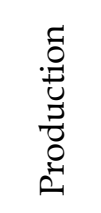 & 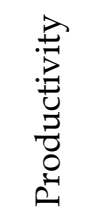 & $\stackrel{\mathbb{2}}{\stackrel{2}{4}}$ & 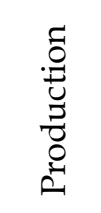 & 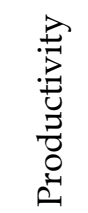 \\
\hline \multirow{7}{*}{ 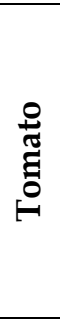 } & $2005-06$ & 12 & 325 & 27.08 & 8 & 329 & 41.13 & 1 & 41 & 41.00 & 21 & 695 & 33.10 \\
\hline & 2006-07 & 12 & 356 & 29.66 & 7 & 281 & 40.14 & 1155 & 55056 & 47.67 & 1174 & 55693 & 47.44 \\
\hline & $2007-08$ & 13 & 481 & 37.00 & 5 & 201 & 40.20 & 306 & 14652 & 47.88 & 324 & 15334 & 47.33 \\
\hline & $2008-09$ & 45 & 1669 & 37.09 & 14 & 563 & 40.21 & 269 & 12885 & 47.90 & 328 & 15117 & 46.09 \\
\hline & $2009-10$ & 76 & 2821 & 37.12 & 14 & 564 & 40.29 & 259 & 12409 & 47.91 & 349 & 15794 & 45.26 \\
\hline & $2010-11$ & 49 & 1839 & 37.53 & 14 & 568 & 40.57 & 378 & 18137 & 47.98 & 441 & 20544 & 46.59 \\
\hline & $2011-12$ & 53 & 1986 & 37.47 & 15 & 613 & 40.87 & 408 & 19588 & 48.01 & 476 & 22187 & 46.61 \\
\hline \multirow{7}{*}{ 点 } & $2005-06$ & 22 & 672 & 30.55 & 18 & 549 & 30.50 & 1 & 67.2 & 67.2 & 41 & 1893 & 46.17 \\
\hline & 2006-07 & 22 & 673 & 30.59 & 21 & 645 & 30.71 & 13 & 673 & 51.8 & 56 & 1991 & 35.55 \\
\hline & 2007-08 & 12 & 367 & 30.58 & 7 & 215 & 30.71 & 9 & 367 & 40.8 & 28 & 949 & 33.89 \\
\hline & 2008-09 & 17 & 520 & 30.59 & 14 & 430 & 30.71 & 6 & 520 & 86.7 & 37 & 1470 & 39.73 \\
\hline & $2009-10$ & 26 & 801 & 30.81 & 10 & 308 & 30.80 & 3 & 80.1 & 26.7 & 39 & 1910 & 48.97 \\
\hline & $2010-11$ & 44 & 1363 & 30.98 & 36 & 1115 & 30.97 & 2 & 136.3 & 68.2 & 82 & 3841 & 46.84 \\
\hline & $2011-12$ & 48 & 1527 & 31.81 & 40 & 1249 & 31.23 & 2 & 152.7 & 76.4 & 90 & 4303 & 47.81 \\
\hline
\end{tabular}




\begin{tabular}{|c|c|c|c|c|c|c|c|c|c|c|c|c|c|}
\hline \multirow[b]{2}{*}{ 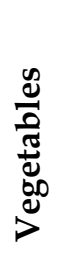 } & \multirow[b]{2}{*}{ 晃 } & \multicolumn{3}{|c|}{ Lalitpur } & \multicolumn{3}{|c|}{ Jhansi } & \multicolumn{3}{|c|}{ Jalaun } & \multicolumn{3}{|c|}{ Jhansi Division } \\
\hline & & 总 & 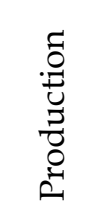 & 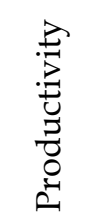 & 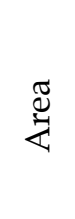 & 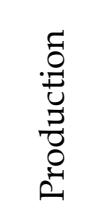 & 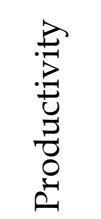 & 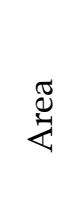 & 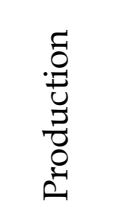 & 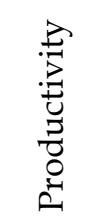 & 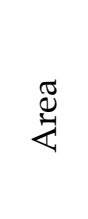 & 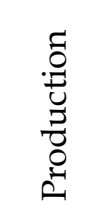 & $\begin{array}{l}\stackrel{D}{0} \\
. \\
0 \\
0 \\
0 \\
0 \\
0 \\
0\end{array}$ \\
\hline \multirow{7}{*}{ 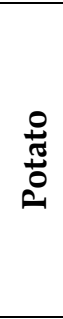 } & 2005-06 & 403 & 9050 & 22.46 & 402 & 9030 & 22.46 & 334 & 7502 & 22.46 & 1139 & 25582 & 22.46 \\
\hline & 2006-07 & 391 & 8617 & 22.04 & 421 & 9279 & 22.04 & 495 & 10910 & 22.04 & 1307 & 28806 & 22.04 \\
\hline & 2007-08 & 214 & 4755 & 22.22 & 322 & 7154 & 22.22 & 411 & 9132 & 22.22 & 947 & 21041 & 22.22 \\
\hline & 2008-09 & 435 & 8937 & 20.55 & 438 & 8999 & 20.55 & 441 & 9061 & 20.55 & 1314 & 26997 & 20.55 \\
\hline & $2009-10$ & 349 & 8652 & 24.79 & 436 & 10809 & 24.79 & 396 & 9818 & 24.79 & 1181 & 29279 & 24.79 \\
\hline & $2010-11$ & 373 & 9008 & 24.15 & 441 & 10650 & 24.15 & 493 & 11905 & 24.15 & 1307 & 31563 & 24.15 \\
\hline & 2011-12 & 517 & 11416 & 22.08 & 642 & 14176 & 22.08 & 597 & 13182 & 22.08 & 1756 & 38774 & 22.08 \\
\hline \multirow{7}{*}{ ○ే } & 2005-06 & 146 & 2059 & 14.10 & 75 & 994 & 13.25 & 155 & 2102 & 13.56 & 376 & 5155 & 13.71 \\
\hline & 2006-07 & 136 & 2543 & 18.70 & 44 & 684 & 15.55 & 96 & 1454 & 15.15 & 276 & 4681 & 16.96 \\
\hline & 2007-08 & 97 & 1048 & 10.80 & 76 & 880 & 11.58 & 94 & 1818 & 19.34 & 267 & 3746 & 14.03 \\
\hline & 2008-09 & 185 & 2190 & 11.84 & 34 & 477 & 14.03 & 195 & 2803 & 14.37 & 414 & 5470 & 13.21 \\
\hline & 2009-10 & 172 & 2875 & 16.72 & 42 & 639 & 15.21 & 525 & 7893 & 15.03 & 739 & 11407 & 15.44 \\
\hline & 2010-11 & 153 & 2682 & 17.53 & 64 & 1000 & 15.63 & 342 & 5043 & 14.75 & 559 & 8725 & 15.61 \\
\hline & 2011-12 & 151 & 3322 & 22.00 & 34 & 512 & 15.06 & 464 & 5617 & 12.11 & 649 & 9451 & 14.56 \\
\hline
\end{tabular}




\begin{tabular}{|c|c|c|c|c|c|c|c|c|c|c|c|c|c|}
\hline \multirow{2}{*}{ 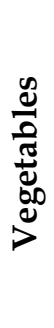 } & \multirow[b]{2}{*}{ 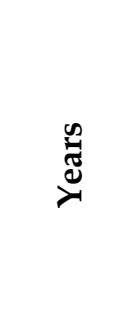 } & \multicolumn{3}{|c|}{ Lalitpur } & \multicolumn{3}{|c|}{ Jhansi } & \multicolumn{3}{|c|}{ Jalaun } & \multicolumn{3}{|c|}{$\begin{array}{c}\text { Jhansi } \\
\text { Division }\end{array}$} \\
\hline & & 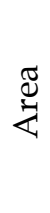 & 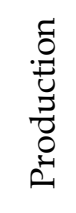 & 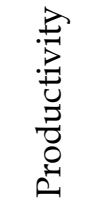 & $\stackrel{\pi}{\mathbb{U}}$ & 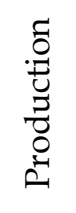 & 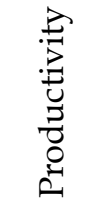 & 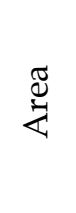 & 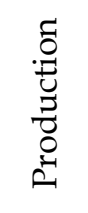 & 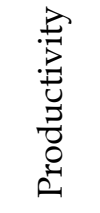 & 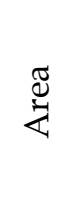 & 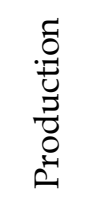 & $\begin{array}{l}\stackrel{D}{D} \\
. \\
0 \\
0 \\
0 \\
0 \\
0 \\
0\end{array}$ \\
\hline \multirow{7}{*}{$\frac{\pi}{0}$} & 2005-06 & 5 & 70 & 14.0 & 60 & 653 & 10.88 & 66 & 925 & 14.02 & 131 & 1648 & 12.58 \\
\hline & 2006-07 & 1 & 14 & 14.0 & 75 & 821 & 10.95 & 3 & 42 & 14.00 & 79 & 877 & 11.10 \\
\hline & 2007-08 & 12 & 168 & 14.0 & 83 & 909 & 10.95 & 20 & 280 & 14.00 & 115 & 1357 & 11.80 \\
\hline & 2008-09 & 23 & 331 & 14.39 & 62 & 670 & 10.81 & 30 & 425 & 14.17 & 115 & 1426 & 12.40 \\
\hline & $2009-10$ & 61 & 879 & 14.41 & 51 & 558 & 10.94 & 309 & 4457 & 14.42 & 421 & 5894 & 14.00 \\
\hline & 2010-11 & 17 & 251 & 14.76 & 55 & 605 & 11.00 & 45 & 670 & 14.89 & 117 & 1526 & 13.04 \\
\hline & 2011-12 & 18 & 266 & 14.78 & 57 & 641 & 11.25 & 47 & 710 & 15.11 & 122 & 1617 & 13.25 \\
\hline \multirow{7}{*}{ 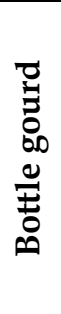 } & 2005-06 & 2 & 76 & 38.0 & 13 & 359 & 27.62 & 13 & 351 & 27.00 & 28 & 786 & 28.07 \\
\hline & 2006-07 & 2 & 79 & 39.5 & 13 & 360 & 27.69 & 18 & 495 & 27.50 & 33 & 934 & 28.30 \\
\hline & 2007-08 & 3 & 83 & 27.67 & 11 & 305 & 27.73 & 31 & 855 & 27.58 & 45 & 1243 & 27.62 \\
\hline & 2008-09 & 3 & 139 & 27.8 & 6 & 166 & 27.67 & 13 & 359 & 27.62 & 22 & 664 & 30.18 \\
\hline & 2009-10 & 5 & 139 & 27.8 & 8 & 223 & 27.88 & 10 & 278 & 27.80 & 23 & 640 & 27.83 \\
\hline & 2010-11 & 5 & 150 & 30.0 & 20 & 565 & 28.25 & 10 & 283 & 28.30 & 35 & 998 & 28.51 \\
\hline & $2011-12$ & 6 & 157 & 26.16 & 22 & 628 & 28.55 & 11 & 314 & 28.55 & 39 & 1099 & 28.18 \\
\hline
\end{tabular}




\begin{tabular}{|c|c|c|c|c|c|c|c|c|c|c|c|c|c|}
\hline \multirow[b]{2}{*}{ 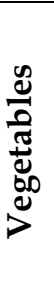 } & \multirow[b]{2}{*}{ 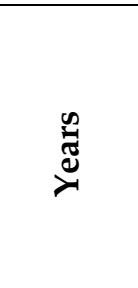 } & \multicolumn{3}{|c|}{ Lalitpur } & \multicolumn{3}{|c|}{ Jhansi } & \multicolumn{3}{|c|}{ Jalaun } & \multicolumn{3}{|c|}{ Jhansi Division } \\
\hline & & $\underset{\Xi}{\stackrel{\Xi}{Z}}$ & 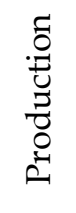 & 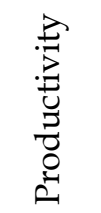 & $\underset{\mathbb{Z}}{\mathbb{Z}}$ & 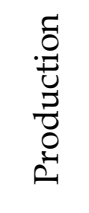 & 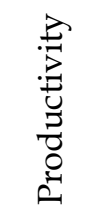 & $\underset{\mathbb{Z}}{\mathbb{Z}}$ & 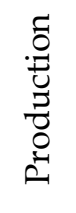 & 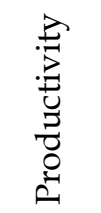 & $\underset{\Xi}{\mathbb{Z}}$ & 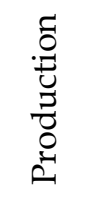 & $\begin{array}{l}\stackrel{2}{D} \\
. \\
0 \\
D \\
0 \\
0 \\
0 \\
=\end{array}$ \\
\hline \multirow{7}{*}{ 茝 } & 2005-06 & 1 & 12 & 12.0 & 11 & 171 & 15.55 & 7 & 109 & 15.57 & 19 & 292 & 15.37 \\
\hline & 2006-07 & 2 & 13 & 6.5 & 3 & 45 & 15.00 & 16 & 257 & 16.06 & 21 & 315 & 15.00 \\
\hline & 2007-08 & 1 & 15 & 15.0 & 2 & 31 & 15.50 & 21 & 338 & 16.10 & 24 & 384 & 16.00 \\
\hline & 2008-09 & 2 & 31 & 15.5 & 8 & 125 & 15.63 & 23 & 372 & 16.17 & 33 & 528 & 16.00 \\
\hline & 2009-10 & 2 & 31 & 15.5 & 3 & 47 & 15.67 & 24 & 398 & 16.58 & 29 & 476 & 16.41 \\
\hline & $2010-11$ & 1 & 18 & 18.0 & 11 & 204 & 18.55 & 21 & 389 & 18.52 & 33 & 611 & 18.52 \\
\hline & 2011-12 & 1 & 18 & 18.0 & 13 & 234 & 18.00 & 24 & 447 & 18.63 & 38 & 699 & 18.39 \\
\hline \multirow{7}{*}{ نั } & $2005-06$ & 26 & 524 & 20.15 & 13 & 263 & 20.23 & 1 & 20 & 20.00 & 40 & 807 & 20.18 \\
\hline & 2006-07 & 32 & 645 & 20.16 & 3 & 61 & 20.33 & 2 & 23 & 11.50 & 37 & 729 & 19.70 \\
\hline & 2007-08 & 7 & 141 & 20.14 & 5 & 101 & 20.20 & 1 & 34 & 34.00 & 13 & 276 & 21.23 \\
\hline & 2008-09 & 25 & 504 & 20.16 & 6 & 122 & 20.33 & 1 & 20 & 20.00 & 32 & 646 & 20.19 \\
\hline & 2009-10 & 39 & 788 & 20.21 & 1 & 20 & 20.00 & 2 & 40 & 20.00 & 42 & 848 & 20.19 \\
\hline & 2010-11 & 15 & 308 & 20.53 & 56 & 1151 & 20.55 & 7 & 144 & 20.57 & 78 & 1603 & 20.55 \\
\hline & 2011-12 & 17 & 351 & 20.65 & 62 & 1280 & 20.65 & 8 & 165 & 20.63 & 87 & 1796 & 20.64 \\
\hline
\end{tabular}

Source: Statistics section, State Horticulture Department, Lucknow (U.P.)

Units: Area in Hectares, Production in Metric Tons, and Productivity in Metric Tons/Hectare 
Table: 2 Annual Growth Rates of Area, Production and Productivity of Selected Vegetables (2006 -07 to 2011-12)

\begin{tabular}{|c|c|c|c|c|c|c|c|c|c|c|c|c|c|}
\hline \multirow[b]{2}{*}{ 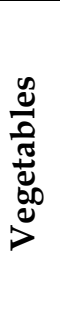 } & \multirow[b]{2}{*}{ 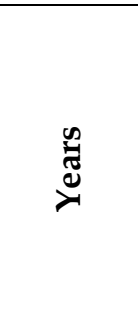 } & \multicolumn{3}{|c|}{ Lalitpur } & \multicolumn{3}{|c|}{ Jhansi } & \multicolumn{3}{|c|}{ Jalaun } & \multicolumn{3}{|c|}{$\begin{array}{c}\text { Jhansi } \\
\text { Division }\end{array}$} \\
\hline & & 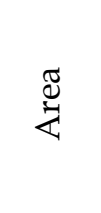 & 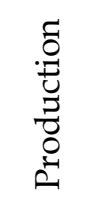 & 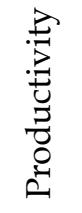 & 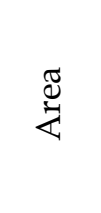 & 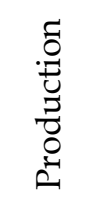 & 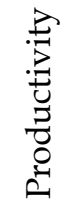 & 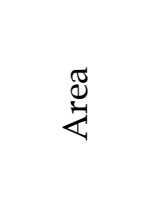 & 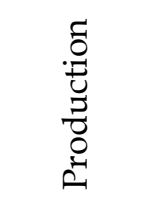 & 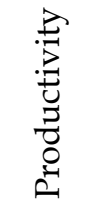 & 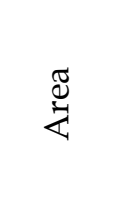 & 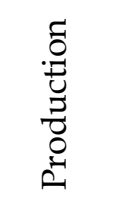 & 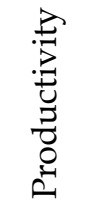 \\
\hline \multirow{6}{*}{ 苞 } & 2006-07 & 0.0 & 9.5 & 9.5 & -12.5 & -14.6 & -2.4 & 115400.0 & 134182.9 & 16.3 & 5490.5 & 7913.4 & 43.3 \\
\hline & 2007-08 & 8.3 & 35.1 & 24.7 & -28.6 & -28.5 & 0.1 & -73.5 & -73.4 & 0.4 & -72.4 & -72.5 & -0.2 \\
\hline & 2008-09 & 246.2 & 247.0 & 0.2 & 180.0 & 180.1 & 0.0 & -12.1 & -12.1 & 0.0 & 1.2 & -1.4 & -2.6 \\
\hline & 2009-10 & 68.9 & 69.0 & 0.1 & 0.0 & 0.2 & 0.2 & -3.7 & -3.7 & 0.0 & 6.4 & 4.5 & -1.8 \\
\hline & $2010-11$ & -35.5 & -34.8 & 1.1 & 0.0 & 0.7 & 0.7 & 45.9 & 46.2 & 0.1 & 26.4 & 30.1 & 2.9 \\
\hline & 2011-12 & 8.2 & 8.0 & -0.2 & 7.1 & 7.9 & 0.7 & 7.9 & 8.0 & 0.1 & 7.9 & 8.0 & 0.0 \\
\hline \multirow{6}{*}{ 元 } & $2006-07$ & 0.0 & 0.1 & 0.1 & 16.7 & 17.5 & 0.7 & 1200.0 & 901.5 & -23.0 & 36.6 & 5.2 & -23.0 \\
\hline & 2007-08 & -45.5 & -45.5 & 0.0 & -66.7 & -66.7 & 0.0 & -30.8 & -45.5 & -21.2 & -50.0 & -52.3 & -4.7 \\
\hline & 2008-09 & 41.7 & 41.7 & 0.0 & 100.0 & 100.0 & 0.0 & -33.3 & 41.7 & 112.5 & 32.1 & 54.9 & 17.2 \\
\hline & 2009-10 & 52.9 & 54.0 & 0.7 & -28.6 & -28.4 & 0.3 & -50.0 & -84.6 & -69.2 & 5.4 & 29.9 & 23.3 \\
\hline & 2010-11 & 69.2 & 70.2 & 0.6 & 260.0 & 262.0 & 0.6 & -33.3 & 70.2 & 155.2 & 110.3 & 101.1 & -4.3 \\
\hline & $2011-12$ & 9.1 & 12.0 & 2.7 & 11.1 & 12.0 & 0.8 & 0.0 & 12.0 & 12.0 & 9.8 & 12.0 & 2.1 \\
\hline
\end{tabular}




\begin{tabular}{|c|c|c|c|c|c|c|c|c|c|c|c|c|c|}
\hline \multirow[b]{2}{*}{ 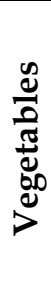 } & \multirow[b]{2}{*}{ 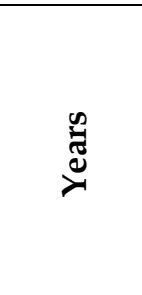 } & \multicolumn{3}{|c|}{ Lalitpur } & \multicolumn{3}{|c|}{ Jhansi } & \multicolumn{3}{|c|}{ Jalaun } & \multicolumn{3}{|c|}{ Jhansi Division } \\
\hline & & 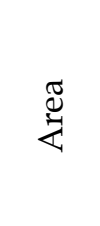 & $\begin{array}{r}0 \\
.0 \\
0 \\
0 \\
0 \\
0 \\
0 \\
0 \\
0\end{array}$ & 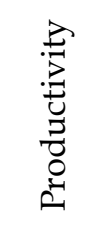 & 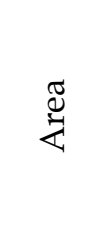 & 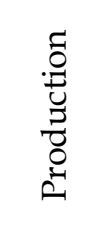 & 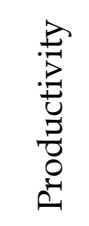 & 党 & 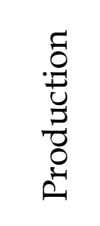 & 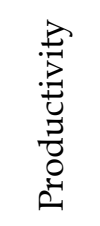 & $\underset{\mathbb{Z}}{\mathbb{Z}}$ & 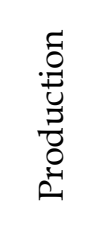 & 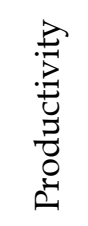 \\
\hline \multirow{6}{*}{$\begin{array}{l}0 \\
\text { O⿱艹 } \\
0 \\
0\end{array}$} & 2006-07 & -3.0 & -4.8 & -1.9 & 4.7 & 2.8 & -1.9 & 48.2 & 45.4 & -1.9 & 14.7 & 12.6 & -1.9 \\
\hline & $2007-08$ & -45.3 & -44.8 & 0.8 & -23.5 & -22.9 & 0.8 & -17.0 & -16.3 & 0.8 & -27.5 & -27.0 & 0.8 \\
\hline & $2008-09$ & 103.3 & 87.9 & -7.5 & 36.0 & 25.8 & -7.5 & 7.3 & -0.8 & -7.5 & 38.8 & 28.3 & -7.5 \\
\hline & $2009-10$ & -19.8 & -3.2 & 20.6 & -0.5 & 20.1 & 20.6 & -10.2 & 8.4 & 20.6 & -10.1 & 8.5 & 20.6 \\
\hline & $2010-11$ & 6.9 & 4.1 & -2.6 & 1.1 & -1.5 & -2.6 & 24.5 & 21.3 & -2.6 & 10.7 & 7.8 & -2.6 \\
\hline & $2011-12$ & 38.6 & 26.7 & -8.6 & 45.6 & 33.1 & -8.6 & 21.1 & 10.7 & -8.6 & 34.4 & 22.8 & -8.6 \\
\hline \multirow{6}{*}{ مี } & 2006-07 & -6.8 & 23.5 & 32.6 & -41.3 & -31.2 & 17.4 & -38.1 & -30.8 & 11.7 & -26.6 & -9.2 & 23.7 \\
\hline & $2007-08$ & -28.7 & -58.8 & -42.2 & 72.7 & 28.7 & -25.5 & -2.1 & 25.0 & 27.7 & -3.3 & -20.0 & -17.3 \\
\hline & 2008-09 & 90.7 & 109.0 & 9.6 & -55.3 & -45.8 & 21.2 & 107.4 & 54.2 & -25.7 & 55.1 & 46.0 & -5.8 \\
\hline & $2009-10$ & -7.0 & 31.3 & 41.2 & 23.5 & 34.0 & 8.4 & 169.2 & 181.6 & 4.6 & 78.5 & 108.5 & 16.9 \\
\hline & $2010-11$ & -11.0 & -6.7 & 4.8 & 52.4 & 56.5 & 2.8 & -34.9 & -36.1 & -1.9 & -24.4 & -23.5 & 1.1 \\
\hline & $2011-12$ & -1.3 & 23.9 & 25.5 & -46.9 & -48.8 & -3.6 & 35.7 & 11.4 & -17.9 & 16.1 & 8.3 & -6.7 \\
\hline
\end{tabular}




\begin{tabular}{|c|c|c|c|c|c|c|c|c|c|c|c|c|c|}
\hline \multirow[b]{2}{*}{ 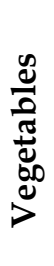 } & \multirow[b]{2}{*}{ 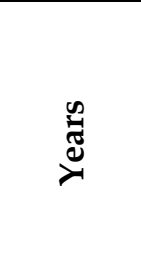 } & \multicolumn{3}{|c|}{ Lalitpur } & \multicolumn{3}{|c|}{ Jhansi } & \multicolumn{3}{|c|}{ Jalaun } & \multicolumn{3}{|c|}{ Jhansi Division } \\
\hline & & 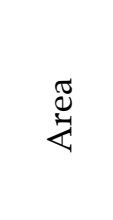 & 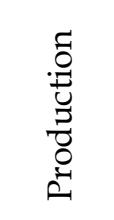 & 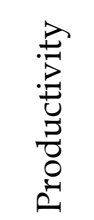 & $\underset{\mathbb{Z}}{\stackrel{\Xi}{Z}}$ & 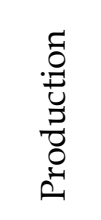 & 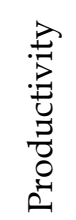 & 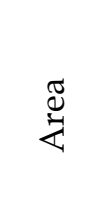 & 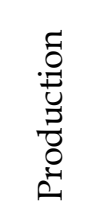 & 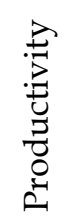 & 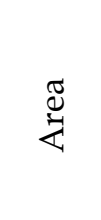 & 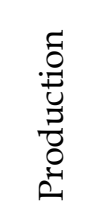 & $\begin{array}{l}\stackrel{D}{2} \\
. \\
0 \\
0 \\
0 \\
0 \\
0 \\
0\end{array}$ \\
\hline \multirow{6}{*}{$\frac{\pi}{0}$} & $2006-07$ & -80.0 & -80.0 & 0.0 & 25.0 & 25.7 & 0.6 & -95.5 & -95.5 & -0.1 & -39.7 & -46.8 & -11.8 \\
\hline & $2007-08$ & 1100.0 & 1100.0 & 0.0 & 10.7 & 10.7 & 0.0 & 566.7 & 566.7 & 0.0 & 45.6 & 54.7 & 6.3 \\
\hline & 2008-09 & 91.7 & 97.0 & 2.8 & -25.3 & -26.3 & -1.3 & 50.0 & 51.8 & 1.2 & 0.0 & 5.1 & 5.1 \\
\hline & $2009-10$ & 165.2 & 165.6 & 0.1 & -17.7 & -16.7 & 1.2 & 930.0 & 948.7 & 1.8 & 266.1 & 313.3 & 12.9 \\
\hline & $2010-11$ & -72.1 & -71.4 & 2.4 & 7.8 & 8.4 & 0.5 & -85.4 & -85.0 & 3.3 & -72.2 & -74.1 & -6.9 \\
\hline & $2011-12$ & 5.9 & 6.0 & 0.1 & 3.6 & 6.0 & 2.3 & 4.4 & 6.0 & 1.5 & 4.3 & 6.0 & 1.6 \\
\hline \multirow{6}{*}{ 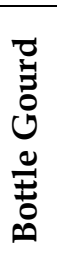 } & $2006-07$ & 0.0 & 3.9 & 3.9 & 0.0 & 0.3 & 0.3 & 38.5 & 41.0 & 1.9 & 17.9 & 18.8 & 0.8 \\
\hline & $2007-08$ & 50.0 & 5.1 & -29.9 & -15.4 & -15.3 & 0.1 & 72.2 & 72.7 & 0.3 & 36.4 & 33.1 & -2.4 \\
\hline & 2008-09 & 0.0 & 67.5 & 0.5 & -45.5 & -45.6 & -0.2 & -58.1 & -58.0 & 0.1 & -51.1 & -46.6 & 9.3 \\
\hline & $2009-10$ & 66.7 & 0.0 & 0.0 & 33.3 & 34.3 & 0.8 & -23.1 & -22.6 & 0.7 & 4.5 & -3.6 & -7.8 \\
\hline & 2010-11 & 0.0 & 7.9 & 7.9 & 150.0 & 153.4 & 1.3 & 0.0 & 1.8 & 1.8 & 52.2 & 55.9 & 2.4 \\
\hline & 2011-12 & 20.0 & 4.7 & -12.8 & 10.0 & 11.2 & 1.1 & 10.0 & 11.0 & 0.9 & 11.4 & 10.1 & -1.2 \\
\hline
\end{tabular}




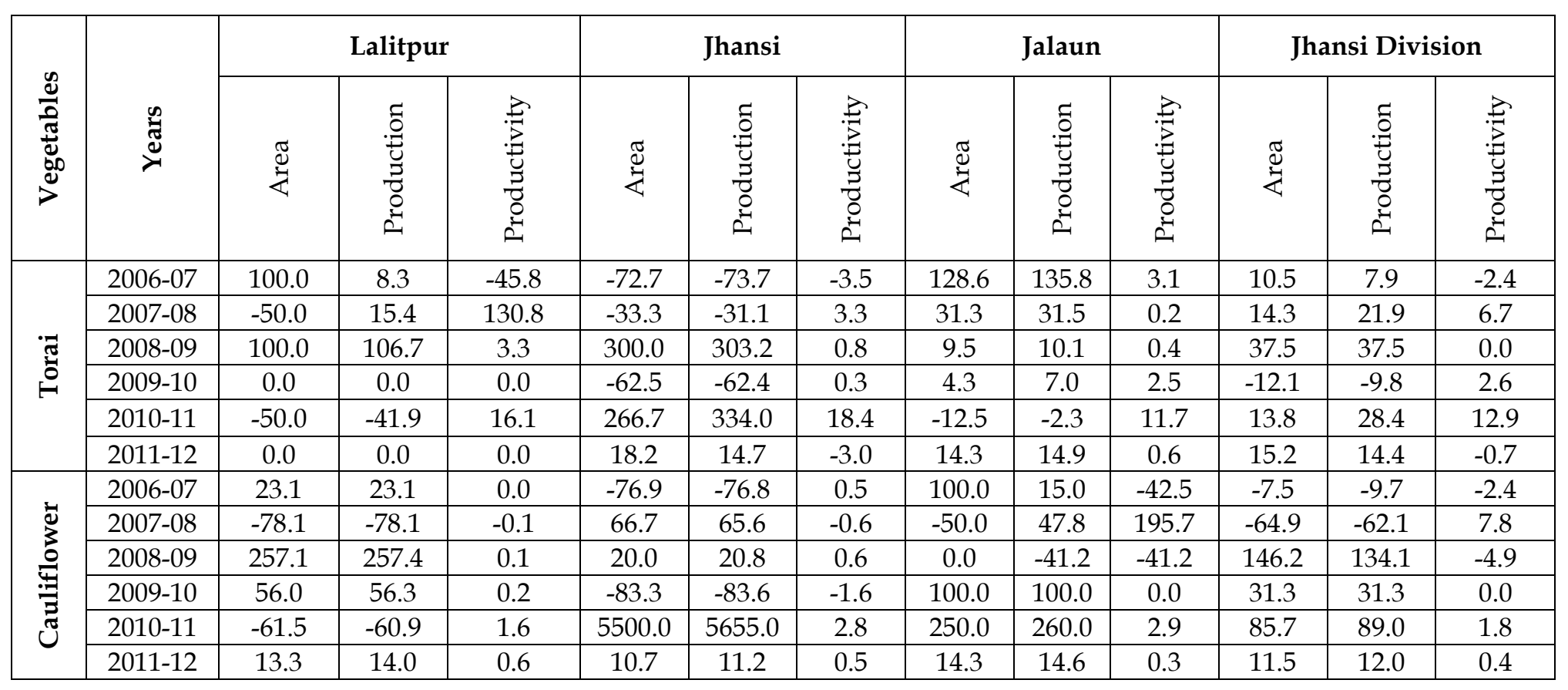

Source: Computed 
Table: 3 Pattern of Arrival and Price of Selected Vegetables in Selected Region

\begin{tabular}{|c|c|c|c|c|c|c|c|c|c|c|c|c|c|}
\hline \multirow[b]{2}{*}{ 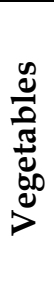 } & \multirow[b]{2}{*}{ む゙ } & \multicolumn{3}{|c|}{ Lalitpur } & \multicolumn{3}{|c|}{ Jhansi } & \multicolumn{3}{|c|}{ Jalaun } & \multicolumn{3}{|c|}{ Jhansi Division } \\
\hline & & 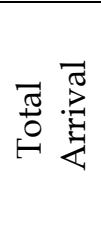 & 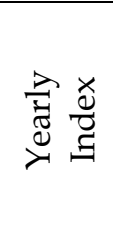 & 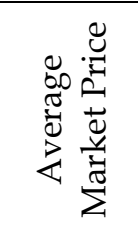 & $\begin{array}{ll}\pi \\
0 \\
0 \\
0\end{array}$ & 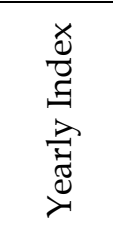 & 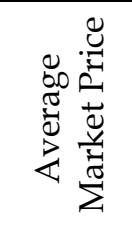 & 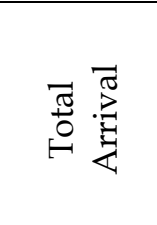 & 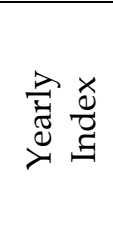 & 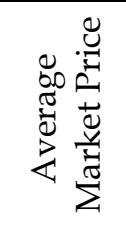 & 苗离 & 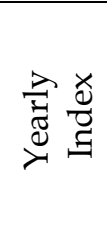 & 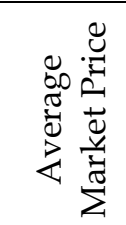 \\
\hline \multirow{8}{*}{ 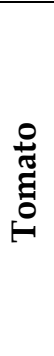 } & 2005 & 3138 & 160.10 & 779.25 & 76010 & 78.02 & 617.75 & NA & - & NA & 79148 & 67.75 & 698.50 \\
\hline & 2006 & 3291 & 167.91 & 714.25 & 84415 & 86.65 & 490.00 & NA & - & NA & 87706 & 75.07 & 602.13 \\
\hline & 2007 & 2677 & 136.58 & 744.67 & 52511 & 53.90 & 698.00 & 840.50 & 2.89 & 1137.33 & 56028 & 47.96 & 860.00 \\
\hline & 2008 & 1166 & 59.49 & 941.83 & 43497 & 44.65 & 789.75 & 2216.10 & 7.62 & 1272.07 & 46879 & 40.13 & 1001.22 \\
\hline & 2009 & 841 & 42.91 & 875.33 & 145692 & 149.55 & 987.99 & 2318.20 & 7.97 & 878.96 & 148851 & 127.41 & 914.09 \\
\hline & 2010 & 829 & 42.30 & 1064.42 & 102195 & 104.90 & 1104.60 & 106138.33 & 365.01 & 988.78 & 209162 & 179.03 & 1052.60 \\
\hline & 2011 & 755 & 38.52 & 998.75 & 209985 & 215.54 & 914.42 & 55302.60 & 190.19 & 885.12 & 266043 & 227.72 & 932.76 \\
\hline & 2012 & 1538 & 78.47 & 1078.08 & 181205 & 186.00 & 929.17 & 7653.90 & 26.32 & 953.46 & 190397 & 162.97 & 986.90 \\
\hline
\end{tabular}




\begin{tabular}{|c|c|c|c|c|c|c|c|c|c|c|c|c|c|}
\hline \multirow[b]{2}{*}{ 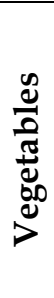 } & \multirow[b]{2}{*}{ 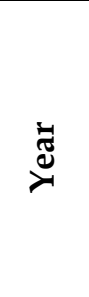 } & \multicolumn{3}{|c|}{ Lalitpur } & \multicolumn{3}{|c|}{ Jhansi } & \multicolumn{3}{|c|}{ Jalaun } & \multicolumn{3}{|c|}{ Jhansi Division } \\
\hline & & 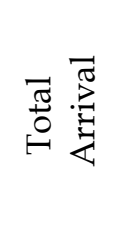 & 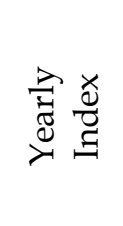 & 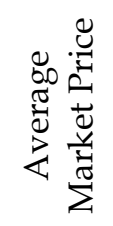 & 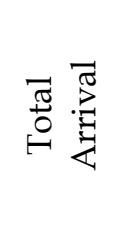 & 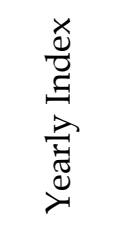 & 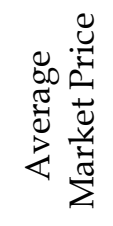 & $\begin{array}{ll}\pi \\
0 \\
0 \\
0\end{array}$ & 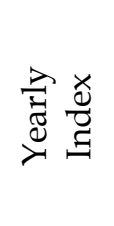 & 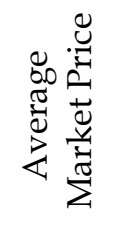 & 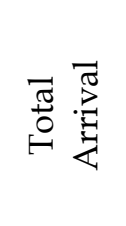 & 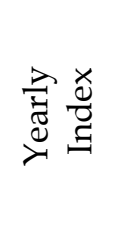 & 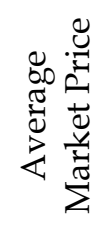 \\
\hline \multirow{8}{*}{ 莡 } & 2005 & 4628 & 179.83 & 521.83 & 14585 & 43.81 & 353.63 & NA & - & NA & 19213 & 48.88 & 437.73 \\
\hline & 2006 & 4018 & 156.12 & 544.67 & 17145 & 51.49 & 417.71 & NA & - & NA & 21163 & 53.84 & 481.19 \\
\hline & 2007 & 2938 & 114.16 & 531.33 & 45222 & 135.82 & 418.08 & 804.80 & 23.42 & 404.62 & 48965 & 124.58 & 451.35 \\
\hline & 2008 & 1409 & 54.75 & 705.58 & 30400 & 91.30 & 641.00 & 1172.60 & 34.13 & 685.67 & 32982 & 83.91 & 677.42 \\
\hline & 2009 & 639 & 24.83 & 628.17 & 35382 & 106.27 & 700.65 & 1512.70 & 44.02 & 655.06 & 37534 & 95.49 & 661.29 \\
\hline & 2010 & 744 & 28.91 & 728.92 & 40365 & 121.23 & 706.70 & 2031.30 & 59.12 & 579.51 & 43140 & 109.76 & 671.71 \\
\hline & 2011 & 662 & 25.72 & 914.50 & 54885 & 164.84 & 745.17 & 9551.50 & 277.97 & 759.87 & 65099 & 165.62 & 806.51 \\
\hline & 2012 & 1029 & 39.98 & 911.33 & 61475 & 184.64 & 799.75 & 5543.90 & 161.34 & 687.30 & 68048 & 173.13 & 799.46 \\
\hline \multirow{8}{*}{ 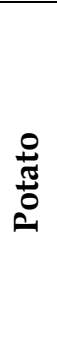 } & 2005 & 79866 & 107.17 & 485.92 & 379547 & 125.02 & 403.25 & NA & - & NA & 459413 & 116.14 & 444.58 \\
\hline & 2006 & 89485 & 120.08 & 585.92 & 273430 & 90.06 & 516.33 & 2037.50 & 8.17 & 611.14 & 364953 & 92.26 & 571.13 \\
\hline & 2007 & 62620 & 84.03 & 592.92 & 241780 & 79.64 & 571.75 & 5913.60 & 23.71 & 584.83 & 310314 & 78.45 & 583.17 \\
\hline & 2008 & 82305 & 110.44 & 361.58 & 315285 & 103.85 & 346.58 & 7367.90 & 29.55 & 374.11 & 404958 & 102.37 & 360.76 \\
\hline & 2009 & 106131 & 142.42 & 807.75 & 339367 & 111.78 & 390.56 & 5571.00 & 22.34 & 833.01 & 451069 & 114.03 & 677.11 \\
\hline & 2010 & 62688 & 84.12 & 498.58 & 363450 & 119.71 & 460.10 & 98154.80 & 393.60 & 531.22 & 524293 & 132.54 & 496.64 \\
\hline & 2011 & 56760 & 76.17 & 496.75 & 461040 & 151.86 & 431.58 & 34272.90 & 137.43 & 430.41 & 552073 & 139.56 & 452.91 \\
\hline & 2012 & 41375 & 55.52 & 847.08 & 404450 & 133.22 & 811.00 & 21246.80 & 85.20 & 818.72 & 467072 & 118.07 & 825.60 \\
\hline
\end{tabular}




\begin{tabular}{|c|c|c|c|c|c|c|c|c|c|c|c|c|c|}
\hline \multirow[b]{2}{*}{$\begin{array}{l}\frac{\infty}{0} \\
\frac{0}{0} \\
\stackrel{0}{0} \\
\stackrel{0}{\infty}\end{array}$} & \multirow[b]{2}{*}{ 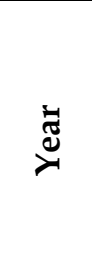 } & \multicolumn{3}{|c|}{ Lalitpur } & \multicolumn{3}{|c|}{ Jhansi } & \multicolumn{3}{|c|}{ Jalaun } & \multicolumn{3}{|c|}{ Jhansi Division } \\
\hline & & 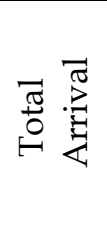 & 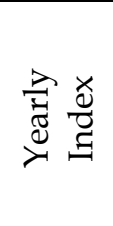 & 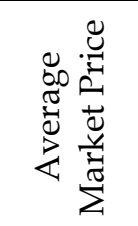 & 完营 & 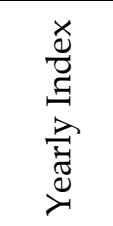 & 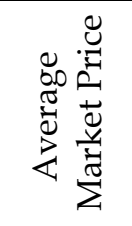 & 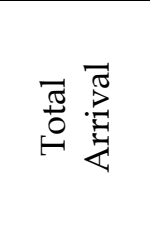 & 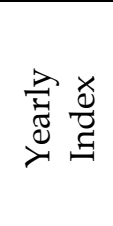 & 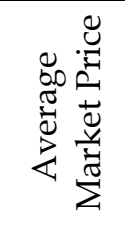 & 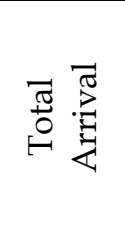 & 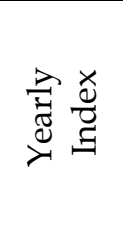 & 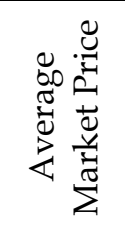 \\
\hline \multirow{8}{*}{ ڤే } & 2005 & 49185 & 128.67 & 585.75 & 96085 & 87.35 & 479.75 & $\mathrm{NA}$ & - & $\mathrm{NA}$ & 145270 & 98.04 & 532.75 \\
\hline & 2006 & 60395 & 158.00 & 414.92 & 91223 & 82.93 & 345.83 & 679.7 & 4.34 & 442.27 & 152298 & 102.78 & 401.01 \\
\hline & 2007 & 41439 & 108.41 & 940.17 & 103978 & 94.53 & 856.67 & 2954.4 & 18.88 & 938.00 & 148371 & 100.13 & 911.61 \\
\hline & 2008 & 51073 & 133.61 & 554.50 & 87190 & 79.27 & 509.92 & 2891.9 & 18.49 & 613.53 & 141155 & 95.26 & 559.32 \\
\hline & 2009 & 14621 & 38.25 & 937.33 & NA & - & NA & 2052.5 & 13.12 & 1042.16 & 16674 & 11.25 & 659.83 \\
\hline & 2010 & 18675 & 48.86 & 952.17 & 126360 & 114.88 & 801.1 & 18346.65 & 117.27 & 801.94 & 163382 & 110.26 & 851.74 \\
\hline & 2011 & 16280 & 42.59 & 971.08 & 146965 & 133.61 & 836.83 & 72386.6 & 462.70 & 868.27 & 235632 & 159.02 & 892.06 \\
\hline & 2012 & 25280 & 66.14 & 692.92 & 155175 & 141.07 & 625.08 & 10198.0 & 65.19 & 625.29 & 190653 & 128.67 & 647.76 \\
\hline \multirow{8}{*}{$\frac{\pi}{0}$} & 2005 & 1268 & 121.57 & 688.17 & 6200 & 53.86 & 1590.67 & NA & - & NA & 7468 & 59.49 & 1139.42 \\
\hline & 2006 & 1368 & 131.16 & 668.88 & 4315 & 37.49 & 682.5 & NA & - & NA & 5683 & 45.27 & 675.69 \\
\hline & 2007 & 1564 & 149.95 & 795.56 & 7405 & 64.33 & 807.2 & NA & - & NA & 8969 & 71.45 & 534.25 \\
\hline & 2008 & 615 & 58.96 & 1749.75 & 7700 & 66.89 & 641.6 & NA & - & NA & 8315 & 66.24 & 797.12 \\
\hline & 2009 & 279 & 26.75 & 1278.00 & 12473 & 108.36 & 1200.76 & NA & - & NA & 12752 & 101.58 & 826.25 \\
\hline & 2010 & 308 & 29.53 & 1164.33 & 17175 & 149.21 & 1329.7 & $\mathrm{NA}$ & - & NA & 17483 & 139.27 & 831.34 \\
\hline & 2011 & 428 & 41.04 & 1452.10 & 23946 & 208.03 & 1115.63 & NA & - & NA & 27374 & 194.16 & 855.91 \\
\hline & 2012 & 589 & 56.47 & 1270.44 & 29952 & 260.21 & 1359.67 & $\mathrm{NA}$ & - & NA & 30541 & 243.28 & 876.70 \\
\hline
\end{tabular}




\begin{tabular}{|c|c|c|c|c|c|c|c|c|c|c|c|c|c|}
\hline \multirow{2}{*}{ 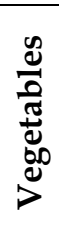 } & \multirow[b]{2}{*}{ ฮ્خ } & \multicolumn{3}{|c|}{ Lalitpur } & \multicolumn{3}{|c|}{ Jhansi } & \multicolumn{3}{|c|}{ Jalaun } & \multicolumn{3}{|c|}{ Jhansi Division } \\
\hline & & 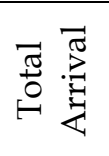 & 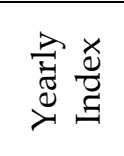 & 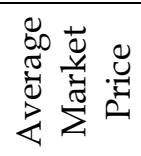 & $\stackrel{\frac{\pi}{0}}{\stackrel{0}{0}}$ & 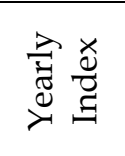 & 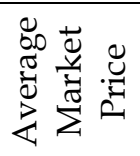 & 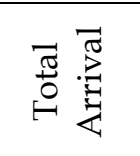 & 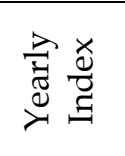 & 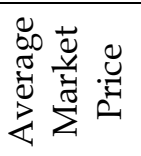 & 焉 & 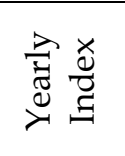 & 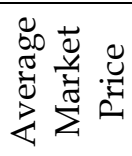 \\
\hline \multirow{8}{*}{ 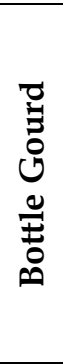 } & 2005 & 3208 & 172.45 & 410.33 & 33430 & 107.32 & 299.6 & NA & - & NA & 36638 & 108.90 & 354.97 \\
\hline & 2006 & 2909 & 156.38 & 443.00 & 41470 & 133.13 & 315.44 & NA & - & NA & 44379 & 131.91 & 379.22 \\
\hline & 2007 & 2270 & 122.03 & 507.50 & 37017 & 118.84 & 350.7 & 551.50 & 52.19 & 477.07 & 39839 & 118.41 & 445.09 \\
\hline & 2008 & 1302 & 69.99 & 708.50 & 30085 & 96.58 & 555.0 & 1083.40 & 102.53 & 709.26 & 32470 & 96.51 & 657.59 \\
\hline & 2009 & 595 & 31.99 & 539.00 & 26685 & 85.67 & 987.88 & 1214.90 & 114.97 & 622.76 & 28495 & 84.70 & 716.55 \\
\hline & 2010 & 709 & 38.11 & 599.25 & 27285 & 87.59 & 1254.0 & 562.10 & 53.19 & 659.07 & 28556 & 84.88 & 837.44 \\
\hline & 2011 & 632 & 33.97 & 748.00 & 36786 & 118.09 & 738.08 & 642.90 & 60.84 & 762.59 & 38061 & 113.13 & 749.56 \\
\hline & 2012 & 1035 & 55.64 & 768.50 & 47850 & 153.61 & 724.17 & 2285.40 & 216.28 & 641.25 & 51170 & 152.09 & 711.31 \\
\hline \multirow{8}{*}{ 节 } & 2005 & 1544 & 145.89 & 768.00 & NA & - & NA & NA & - & NA & 1544.00 & 32.09 & 768.00 \\
\hline & 2006 & 1465 & 138.43 & 690.43 & NA & - & NA & NA & - & NA & 1465.00 & 30.45 & 690.43 \\
\hline & 2007 & 1341 & 126.71 & 533.29 & NA & - & NA & NA & - & NA & 1341.00 & 27.87 & 533.29 \\
\hline & 2008 & 581 & 54.90 & 940.57 & NA & - & NA & NA & - & NA & 581.00 & 12.08 & 940.57 \\
\hline & 2009 & 318 & 30.05 & 903.86 & NA & - & NA & NA & - & NA & 318.00 & 6.61 & 903.86 \\
\hline & 2010 & 296 & 27.97 & 918.00 & 3645 & 48.60 & 1220.0 & 10.80 & 388.49 & 932.67 & 3951.80 & 82.14 & 1023.56 \\
\hline & 2011 & 425 & 40.16 & 1284.11 & 9735 & 129.81 & 1197.57 & 3.10 & 111.51 & 830.16 & 10163.10 & 211.26 & 1103.95 \\
\hline & 2012 & 963 & 90.99 & 1492.75 & 16374 & 218.34 & 1356.44 & 13.90 & 500.00 & 881.41 & 17350.90 & 360.67 & 1243.54 \\
\hline
\end{tabular}




\begin{tabular}{|c|c|c|c|c|c|c|c|c|c|c|c|c|c|}
\hline \multirow{2}{*}{ 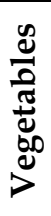 } & \multirow[b]{2}{*}{$\underset{\succsim}{\beth}$} & \multicolumn{3}{|c|}{ Lalitpur } & \multicolumn{3}{|c|}{ Jhansi } & \multicolumn{3}{|c|}{ Jalaun } & \multicolumn{3}{|c|}{ Jhansi Division } \\
\hline & & 중 & 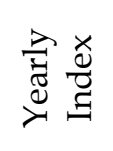 & 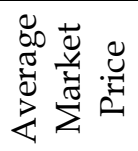 & 坖疍 & 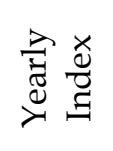 & 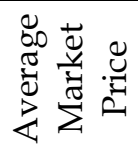 & 坖 & 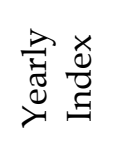 & 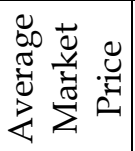 & 푤 & $\begin{array}{l}\vec{\Xi} \\
\stackrel{\Xi}{\Xi} \\
\stackrel{્}{\Xi}\end{array}$ & 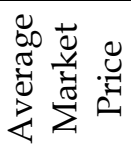 \\
\hline \multirow{8}{*}{$\frac{\bar{d}}{3}$} & 2005 & 2657 & 217.31 & 530.50 & 29990 & 128.67 & 410.4 & NA & - & NA & 32647.00 & 132.84 & 470.45 \\
\hline & 2006 & 2230 & 182.38 & 585.43 & 6556 & 28.13 & 316.33 & NA & - & NA & 8786.00 & 35.75 & 450.88 \\
\hline & 2007 & 790 & 64.61 & 592.25 & 23087 & 99.05 & 533.8 & NA & - & NA & 23877.00 & 97.15 & 563.03 \\
\hline & 2008 & 789 & 64.53 & 1155.20 & 22373 & 95.99 & 521.0 & NA & - & NA & 23162.00 & 94.24 & 838.10 \\
\hline & 2009 & 467 & 38.19 & 958.67 & 21186 & 90.90 & 768.55 & NA & - & NA & 21653.00 & 88.10 & 863.61 \\
\hline & 2010 & 462 & 37.79 & 1158.30 & 20000 & 85.81 & 1267.43 & 5.5 & 3.58 & 427.27 & 20467.50 & 83.28 & 951.00 \\
\hline & 2011 & 571 & 46.70 & 1215.58 & 43015 & 184.55 & 1154.8 & 299.3 & 195.07 & 376.44 & 43885.30 & 178.56 & 915.61 \\
\hline & 2012 & 690 & 56.43 & 1139.63 & 44940 & 192.81 & 1318.5 & 155.5 & 101.35 & 872.66 & 45785.50 & 186.29 & 1110.26 \\
\hline
\end{tabular}

Source: Department of Agriculture Marketing, NavinGallaMandiof Jhansi, Lalitpur and Jalaun (U.P.); Unit: Arrival in Quintal, Price in Rupees; NA: Not available 
Table: 4 Total Production, Retention and Marketed Surplus of Selected Vegetables (Unit: In quintal)

\begin{tabular}{|c|c|c|c|c|c|c|c|c|c|c|c|c|c|c|c|c|c|}
\hline \multirow{3}{*}{ 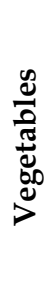 } & \multirow{3}{*}{ 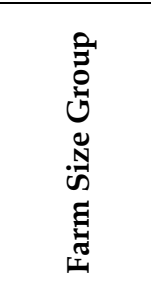 } & \multicolumn{4}{|c|}{ Total Production } & \multicolumn{4}{|c|}{ Marketed Quantity } & \multicolumn{4}{|c|}{ Retention } & \multicolumn{4}{|c|}{ Total No. of Farmers } \\
\hline & & 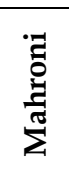 & 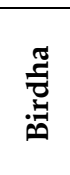 & 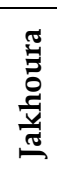 & 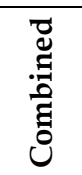 & 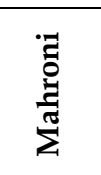 & 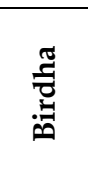 & 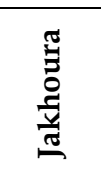 & 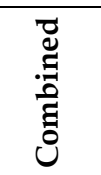 & 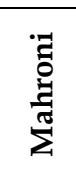 & 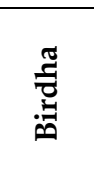 & 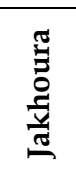 & 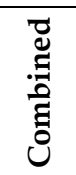 & 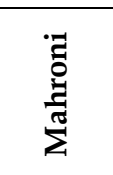 & 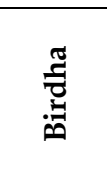 & 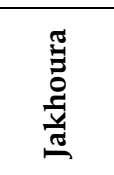 & 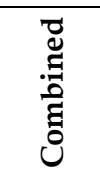 \\
\hline & & $\Xi$ & త & (2) & ت & (6) & e & $E$ & क्ठ & $\approx$ & 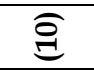 & $\Xi$ & I & 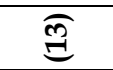 & 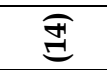 & $\stackrel{\mathscr{2}}{\varrho}$ & $\stackrel{6}{e}$ \\
\hline \multirow{3}{*}{ 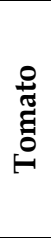 } & Marginal & 328 & 360 & 41 & 729 & $\begin{array}{c}321 \\
(97.9)\end{array}$ & $\begin{array}{c}342 \\
(95.0) \\
\end{array}$ & $\begin{array}{c}39.5 \\
(96.3) \\
\end{array}$ & $\begin{array}{l}702.5 \\
(96.4)\end{array}$ & $\begin{array}{c}7 \\
(2.1) \\
\end{array}$ & $\begin{array}{c}18 \\
(5.0) \\
\end{array}$ & $\begin{array}{c}1.5 \\
(3.7) \\
\end{array}$ & $\begin{array}{l}26.5 \\
(3.6) \\
\end{array}$ & $\begin{array}{c}4 \\
(100.0)\end{array}$ & $\begin{array}{c}6 \\
(100.0) \\
\end{array}$ & $\begin{array}{c}2 \\
(100.0)\end{array}$ & $\begin{array}{c}12 \\
(100.0)\end{array}$ \\
\hline & Small & 680 & 70 & 150 & 900 & $\begin{array}{c}672 \\
(98.8) \\
\end{array}$ & $\begin{array}{c}62 \\
(88.6) \\
\end{array}$ & $\begin{array}{c}147 \\
(98.0) \\
\end{array}$ & $\begin{array}{c}881 \\
(97.9) \\
\end{array}$ & $\begin{array}{c}8 \\
(1.2) \\
\end{array}$ & $\begin{array}{c}8 \\
(11.4) \\
\end{array}$ & $\begin{array}{c}3 \\
(2.0) \\
\end{array}$ & $\begin{array}{c}19 \\
(2.1) \\
\end{array}$ & $\begin{array}{c}5 \\
(100.0) \\
\end{array}$ & $\begin{array}{c}2 \\
(100.0) \\
\end{array}$ & $\begin{array}{c}2 \\
(100.0) \\
\end{array}$ & $\begin{array}{c}9 \\
(100.0)\end{array}$ \\
\hline & Large & 300 & 395 & 330 & 1025 & $\begin{array}{c}294 \\
(98.0)\end{array}$ & $\begin{array}{c}377 \\
(95.4) \\
\end{array}$ & $\begin{array}{c}311 \\
(94.2)\end{array}$ & $\begin{array}{c}982 \\
(95.8)\end{array}$ & $\begin{array}{c}6 \\
(2.0) \\
\end{array}$ & $\begin{array}{c}18 \\
(4.6) \\
\end{array}$ & $\begin{array}{c}19 \\
(5.8) \\
\end{array}$ & $\begin{array}{c}43 \\
(4.2) \\
\end{array}$ & $\begin{array}{c}4 \\
(100.0)\end{array}$ & $\begin{array}{c}8 \\
(100.0) \\
\end{array}$ & $\begin{array}{c}2 \\
(100.0)\end{array}$ & $\begin{array}{c}14 \\
(100.0)\end{array}$ \\
\hline \multirow{3}{*}{ 黑 } & Marginal & 266 & 368 & 488 & 1122 & $\begin{array}{c}257 \\
(96.6)\end{array}$ & $\begin{array}{c}355 \\
(96.5) \\
\end{array}$ & $\begin{array}{c}485 \\
(99.4)\end{array}$ & $\begin{array}{l}1097 \\
(97.8)\end{array}$ & $\begin{array}{c}9 \\
(3.4)\end{array}$ & $\begin{array}{c}13 \\
(3.5)\end{array}$ & $\begin{array}{c}3 \\
(0.6)\end{array}$ & $\begin{array}{c}25 \\
(2.2)\end{array}$ & $\begin{array}{c}5 \\
(100.0)\end{array}$ & $\begin{array}{c}6 \\
(100.0)\end{array}$ & $\begin{array}{c}2 \\
(100.0)\end{array}$ & $\begin{array}{c}13 \\
(100.0)\end{array}$ \\
\hline & Small & 540 & 238 & 92 & 870 & $\begin{array}{c}526 \\
(97.4)\end{array}$ & $\begin{array}{c}222 \\
(93.3) \\
\end{array}$ & $\begin{array}{c}89 \\
(96.7)\end{array}$ & $\begin{array}{c}837 \\
(96.2)\end{array}$ & $\begin{array}{c}14 \\
(2.6)\end{array}$ & $\begin{array}{c}16 \\
(6.7) \\
\end{array}$ & $\begin{array}{c}3 \\
(3.3) \\
\end{array}$ & $\begin{array}{c}33 \\
(3.8) \\
\end{array}$ & $\begin{array}{c}5 \\
(100.0)\end{array}$ & $\begin{array}{c}4 \\
(100.0)\end{array}$ & $\begin{array}{c}2 \\
(100.0)\end{array}$ & $\begin{array}{c}11 \\
(100.0)\end{array}$ \\
\hline & Large & 380 & 888 & 221 & 1489 & $\begin{array}{c}355 \\
(96.5)\end{array}$ & $\begin{array}{c}866 \\
(97.5) \\
\end{array}$ & $\begin{array}{c}213 \\
(96.4)\end{array}$ & $\begin{array}{c}1442 \\
(96.8)\end{array}$ & $\begin{array}{c}18 \\
(4.7)\end{array}$ & $\begin{array}{c}22 \\
(2.5)\end{array}$ & $\begin{array}{c}8 \\
(3.6) \\
\end{array}$ & $\begin{array}{c}48 \\
(3.2)\end{array}$ & $\begin{array}{c}8 \\
(100.0)\end{array}$ & $\begin{array}{c}7 \\
(100.0)\end{array}$ & $\begin{array}{c}4 \\
(100.0)\end{array}$ & $\begin{array}{c}19 \\
(100.0)\end{array}$ \\
\hline
\end{tabular}




\begin{tabular}{|c|c|c|c|c|c|c|c|c|c|c|c|c|c|c|c|c|c|}
\hline \multirow{3}{*}{ 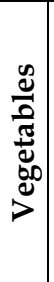 } & \multirow{3}{*}{ 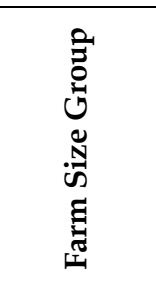 } & \multicolumn{4}{|c|}{ Total Production } & \multicolumn{4}{|c|}{ Marketed Quantity } & \multicolumn{4}{|c|}{ Retention } & \multicolumn{4}{|c|}{ Total No. of Farmers } \\
\hline & & 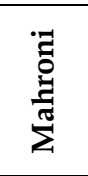 & 苛 & 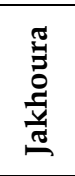 & 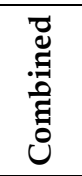 & 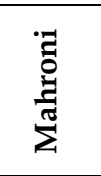 & 胥 & 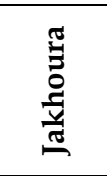 & 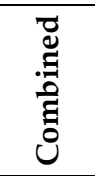 & 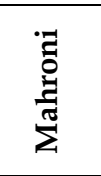 & 孠 & 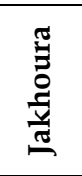 & 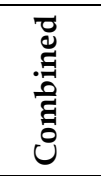 & 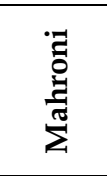 & 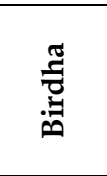 & 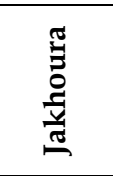 & 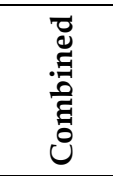 \\
\hline & & $\Xi$ & త & (ิ) & F & (ื) & e & $E$ & क्ठ & $\approx$ & อ & $\Xi$ & త্ & $\stackrel{\widehat{n}}{=}$ & $\overparen{E}$ & $\stackrel{\sqrt[12]{2}}{2}$ & $\stackrel{6}{2}$ \\
\hline \multirow{3}{*}{ 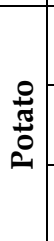 } & Marginal & 470 & 1050 & 390 & 1910 & $\begin{array}{c}445 \\
(94.7)\end{array}$ & $\begin{array}{c}990 \\
(94.3)\end{array}$ & $\begin{array}{c}381 \\
(97.7)\end{array}$ & $\begin{array}{c}1816 \\
(95.1)\end{array}$ & $\begin{array}{c}25 \\
(5.3)\end{array}$ & $\begin{array}{c}60 \\
(5.7)\end{array}$ & $\begin{array}{c}9 \\
(2.3)\end{array}$ & $\begin{array}{c}94 \\
(4.9)\end{array}$ & $\begin{array}{c}2 \\
(100.0)\end{array}$ & $\begin{array}{c}4 \\
(100.0)\end{array}$ & $\begin{array}{c}3 \\
(100.0)\end{array}$ & $\begin{array}{c}9 \\
(100.00)\end{array}$ \\
\hline & Small & 610 & 1135 & 155 & 1900 & $\begin{array}{c}577 \\
(94.6)\end{array}$ & $\begin{array}{l}1101 \\
(97.0)\end{array}$ & $\begin{array}{c}148 \\
(95.5)\end{array}$ & $\begin{array}{c}1826 \\
(96.1)\end{array}$ & $\begin{array}{c}33 \\
(5.4)\end{array}$ & $\begin{array}{c}34 \\
(3.0)\end{array}$ & $\begin{array}{c}7 \\
(4.5)\end{array}$ & $\begin{array}{c}74 \\
(3.9)\end{array}$ & $\begin{array}{c}4 \\
(100.0)\end{array}$ & $\begin{array}{c}5 \\
(100.0)\end{array}$ & $\begin{array}{c}2 \\
(100.0)\end{array}$ & $\begin{array}{c}11 \\
(100.00)\end{array}$ \\
\hline & Large & 1360 & 2070 & 445 & 3875 & $\begin{array}{l}1320 \\
(97.1)\end{array}$ & $\begin{array}{c}2003 \\
(96.8)\end{array}$ & $\begin{array}{c}428 \\
(96.2)\end{array}$ & $\begin{array}{c}3751 \\
(96.8)\end{array}$ & $\begin{array}{c}40 \\
(2.9)\end{array}$ & $\begin{array}{c}67 \\
(3.2) \\
\end{array}$ & $\begin{array}{c}17 \\
(3.8)\end{array}$ & $\begin{array}{c}124 \\
(3.2) \\
\end{array}$ & $\begin{array}{c}8 \\
(100.0)\end{array}$ & $\begin{array}{c}9 \\
(100.0)\end{array}$ & $\begin{array}{c}4 \\
(100.0)\end{array}$ & $\begin{array}{c}21 \\
(100.0)\end{array}$ \\
\hline \multirow{3}{*}{ 莞 } & Marginal & 120 & 167 & 30 & 317 & $\begin{array}{c}119 \\
(99.2)\end{array}$ & $\begin{array}{c}160 \\
(95.8)\end{array}$ & $\begin{array}{c}29 \\
(96.7)\end{array}$ & $\begin{array}{c}308 \\
(97.2)\end{array}$ & $\begin{array}{c}1 \\
(0.8)\end{array}$ & $\begin{array}{c}7 \\
(4.2) \\
\end{array}$ & $\begin{array}{c}1 \\
(3.3)\end{array}$ & $\begin{array}{c}9 \\
(2.8)\end{array}$ & $\begin{array}{c}1 \\
(100.0)\end{array}$ & $\begin{array}{c}3 \\
(100.0)\end{array}$ & $\begin{array}{c}1 \\
(100.0)\end{array}$ & $\begin{array}{c}5 \\
(100.0)\end{array}$ \\
\hline & Small & 45 & 250 & 77 & 372 & $\begin{array}{c}40 \\
(88.9)\end{array}$ & $\begin{array}{c}210 \\
(84.0)\end{array}$ & $\begin{array}{c}74 \\
(96.1)\end{array}$ & $\begin{array}{c}324 \\
(87.1)\end{array}$ & $\begin{array}{c}5 \\
(11.1)\end{array}$ & $\begin{array}{c}40 \\
(16.0)\end{array}$ & $\begin{array}{c}3 \\
(3.9)\end{array}$ & $\begin{array}{c}48 \\
(12.9)\end{array}$ & $\begin{array}{c}2 \\
(100.0)\end{array}$ & $\begin{array}{c}4 \\
(100.0)\end{array}$ & $\begin{array}{c}2 \\
(100.0)\end{array}$ & $\begin{array}{c}8 \\
(100.0)\end{array}$ \\
\hline & Large & 149 & 197 & 247 & 593 & $\begin{array}{c}136 \\
(91.3)\end{array}$ & $\begin{array}{c}180 \\
(91.4)\end{array}$ & $\begin{array}{c}238 \\
(96.4)\end{array}$ & $\begin{array}{c}554 \\
(93.4)\end{array}$ & $\begin{array}{c}13 \\
(8.7)\end{array}$ & $\begin{array}{c}17 \\
(8.6)\end{array}$ & $\begin{array}{c}9 \\
(3.6)\end{array}$ & $\begin{array}{c}39 \\
(6.6)\end{array}$ & $\begin{array}{c}6 \\
(100.0)\end{array}$ & $\begin{array}{c}6 \\
(100.0)\end{array}$ & $\begin{array}{c}5 \\
(100.0)\end{array}$ & $\begin{array}{c}17 \\
(100.0)\end{array}$ \\
\hline \multirow{3}{*}{ 这 } & Marginal & 110 & 152 & 40 & 302 & $\begin{array}{c}109 \\
(99.1)\end{array}$ & $\begin{array}{c}150 \\
(98.7)\end{array}$ & $\begin{array}{c}39 \\
(97.5)\end{array}$ & $\begin{array}{c}298 \\
(98.7)\end{array}$ & $\begin{array}{c}1 \\
(0.9)\end{array}$ & $\begin{array}{c}2 \\
(1.3)\end{array}$ & $\begin{array}{c}1 \\
(2.5)\end{array}$ & $\begin{array}{c}4 \\
(1.3)\end{array}$ & $\begin{array}{c}1 \\
(100.0)\end{array}$ & $\begin{array}{c}2 \\
(100.0)\end{array}$ & $\begin{array}{c}1 \\
(100.0)\end{array}$ & $\begin{array}{c}4 \\
(100.0)\end{array}$ \\
\hline & Small & 185 & 0 & 24 & 209 & $\begin{array}{c}178 \\
(96.2)\end{array}$ & $\begin{array}{c}0 \\
(0.0)\end{array}$ & $\begin{array}{c}20 \\
(83.3)\end{array}$ & $\begin{array}{c}198 \\
(94.7)\end{array}$ & $\begin{array}{c}7 \\
(3.8)\end{array}$ & $\begin{array}{c}(1.0) \\
(0.0)\end{array}$ & $\begin{array}{c}4 \\
(16.7)\end{array}$ & $\begin{array}{c}11 \\
(5.3)\end{array}$ & $\begin{array}{c}6 \\
(100.0)\end{array}$ & $\begin{array}{c}0.0 \\
(0.0)\end{array}$ & $\begin{array}{c}2 \\
(100.0)\end{array}$ & $\begin{array}{c}8 \\
(100.0)\end{array}$ \\
\hline & Large & 410 & 32 & 90 & 532 & $\begin{array}{c}403 \\
(98.3)\end{array}$ & $\begin{array}{c}29 \\
(90.0)\end{array}$ & $\begin{array}{c}90 \\
(100.0)\end{array}$ & $\begin{array}{c}522 \\
(98.1)\end{array}$ & $\begin{array}{c}7 \\
(1.7)\end{array}$ & $\begin{array}{c}3 \\
(9.4)\end{array}$ & $\begin{array}{c}0 \\
(0.0)\end{array}$ & $\begin{array}{c}10 \\
(1.9)\end{array}$ & $\begin{array}{c}6 \\
(100.0)\end{array}$ & $\begin{array}{c}1 \\
(100.0)\end{array}$ & $\begin{array}{c}2 \\
(100.0)\end{array}$ & $\begin{array}{c}10 \\
(100.0)\end{array}$ \\
\hline
\end{tabular}




\begin{tabular}{|c|c|c|c|c|c|c|c|c|c|c|c|c|c|c|c|c|c|}
\hline \multirow{3}{*}{ 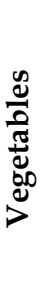 } & \multirow{3}{*}{ 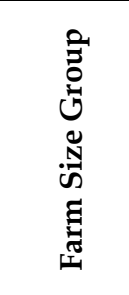 } & \multicolumn{4}{|c|}{ Total Production } & \multicolumn{4}{|c|}{ Marketed Quantity } & \multicolumn{4}{|c|}{ Retention } & \multicolumn{4}{|c|}{ Total No. of Farmers } \\
\hline & & 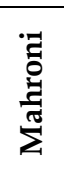 & 疋 & 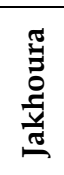 & 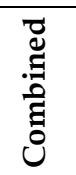 & 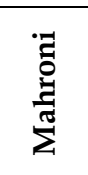 & 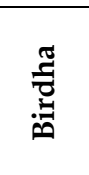 & 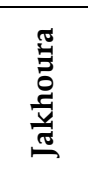 & 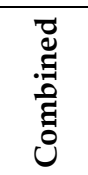 & 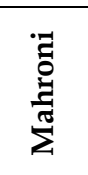 & 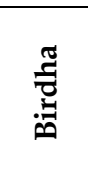 & 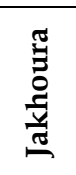 & 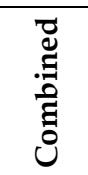 & 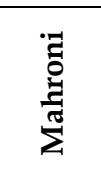 & 疋 & 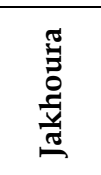 & 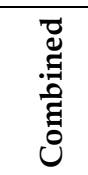 \\
\hline & & $\Xi$ & $\overparen{d}$ & क्ष & F & อี & e & $E$ & $\widetilde{\infty}$ & ฮ & $\stackrel{\widehat{\rho}}{\varrho}$ & $\widetilde{\Xi}$ & $\overparen{\mathfrak{I}}$ & $\stackrel{\overparen{3}}{\varrho}$ & $\underset{\Xi}{\mathbb{E}}$ & $\stackrel{10}{\varrho}$ & $\stackrel{6}{e}$ \\
\hline \multirow{3}{*}{ 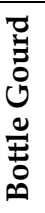 } & Marginal & 290 & 200 & 82 & 572 & $\begin{array}{c}283 \\
(97.6)\end{array}$ & $\begin{array}{c}79 \\
(39.5)\end{array}$ & $\begin{array}{c}80 \\
(97.6)\end{array}$ & $\begin{array}{c}442 \\
(97.8)\end{array}$ & $\begin{array}{c}7 \\
(2.4)\end{array}$ & $\begin{array}{c}121 \\
(60.5)\end{array}$ & $\begin{array}{c}2 \\
(2.4)\end{array}$ & $\begin{array}{c}130 \\
(22.7)\end{array}$ & $\begin{array}{c}4 \\
(100.0)\end{array}$ & $\begin{array}{c}2 \\
(100.0)\end{array}$ & $\begin{array}{c}1 \\
(100.0)\end{array}$ & $\begin{array}{c}7 \\
(100.0)\end{array}$ \\
\hline & Small & 510 & 147 & 170 & 827 & $\begin{array}{c}406 \\
(79.6) \\
\end{array}$ & $\begin{array}{c}144 \\
(98.0) \\
\end{array}$ & $\begin{array}{c}164 \\
(96.5) \\
\end{array}$ & $\begin{array}{c}714 \\
(86.3) \\
\end{array}$ & $\begin{array}{c}104 \\
(20.4) \\
\end{array}$ & $\begin{array}{c}3 \\
(2.0) \\
\end{array}$ & $\begin{array}{c}6 \\
(3.5) \\
\end{array}$ & $\begin{array}{c}113 \\
(13.7) \\
\end{array}$ & $\begin{array}{c}4 \\
(100.0) \\
\end{array}$ & $\begin{array}{c}2 \\
(100.0) \\
\end{array}$ & $\begin{array}{c}3 \\
(100.0)\end{array}$ & $\begin{array}{c}9 \\
(100.0) \\
\end{array}$ \\
\hline & Large & 410 & 660 & 760 & 1830 & $\begin{array}{c}382 \\
(93.2) \\
\end{array}$ & $\begin{array}{c}640 \\
(97.0) \\
\end{array}$ & $\begin{array}{c}748 \\
(98.4) \\
\end{array}$ & $\begin{array}{l}1770 \\
(96.7) \\
\end{array}$ & $\begin{array}{c}28 \\
(6.8) \\
\end{array}$ & $\begin{array}{c}20 \\
(3.0) \\
\end{array}$ & $\begin{array}{c}12 \\
(1.6)\end{array}$ & $\begin{array}{c}60 \\
(3.3) \\
\end{array}$ & $\begin{array}{c}5 \\
(100.0)\end{array}$ & $\begin{array}{c}7 \\
(100.0)\end{array}$ & $\begin{array}{c}2 \\
(100.0)\end{array}$ & $\begin{array}{c}14 \\
(100.0) \\
\end{array}$ \\
\hline \multirow{3}{*}{ 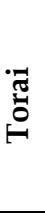 } & Marginal & 68 & 187 & 50 & 305 & $\begin{array}{c}67 \\
(98.5) \\
\end{array}$ & $\begin{array}{c}181 \\
(96.8) \\
\end{array}$ & $\begin{array}{c}49 \\
(98.0) \\
\end{array}$ & $\begin{array}{c}297 \\
(97.4) \\
\end{array}$ & $\begin{array}{c}1 \\
(1.5) \\
\end{array}$ & $\begin{array}{c}6 \\
(3.2) \\
\end{array}$ & $\begin{array}{c}1 \\
(2.0) \\
\end{array}$ & $\begin{array}{c}8 \\
(2.6) \\
\end{array}$ & $\begin{array}{c}1 \\
(100.0) \\
\end{array}$ & $\begin{array}{c}4 \\
(100.0) \\
\end{array}$ & $\begin{array}{c}1 \\
(100.0) \\
\end{array}$ & $\begin{array}{c}6 \\
(100.0) \\
\end{array}$ \\
\hline & Small & 210 & 72 & 0 & 282 & $\begin{array}{c}205 \\
(97.6)\end{array}$ & $\begin{array}{c}70 \\
(97.2) \\
\end{array}$ & $\begin{array}{c}0 \\
(0.0)\end{array}$ & $\begin{array}{c}275 \\
(97.5) \\
\end{array}$ & $\begin{array}{c}5 \\
(2.4) \\
\end{array}$ & $\begin{array}{c}2 \\
(2.8) \\
\end{array}$ & $\begin{array}{c}0 \\
(0.0)\end{array}$ & $\begin{array}{c}7 \\
(2.5) \\
\end{array}$ & $\begin{array}{c}3 \\
(100.0)\end{array}$ & $\begin{array}{c}2 \\
(100.0)\end{array}$ & $\begin{array}{c}0 \\
(0.0)\end{array}$ & $\begin{array}{c}5 \\
(100.0) \\
\end{array}$ \\
\hline & Large & 132 & 210 & 0 & 342 & $\begin{array}{c}124 \\
(93.9) \\
\end{array}$ & $\begin{array}{c}201 \\
(95.7) \\
\end{array}$ & $\begin{array}{c}0 \\
(0.0) \\
\end{array}$ & $\begin{array}{c}325 \\
(95.0) \\
\end{array}$ & $\begin{array}{c}8 \\
(6.1) \\
\end{array}$ & $\begin{array}{c}9 \\
(4.3) \\
\end{array}$ & $\begin{array}{c}0 \\
(0.0)\end{array}$ & $\begin{array}{c}17 \\
(5.0) \\
\end{array}$ & $\begin{array}{c}5 \\
(100.0)\end{array}$ & $\begin{array}{c}6 \\
(100.0)\end{array}$ & $\begin{array}{c}0 \\
(0.0)\end{array}$ & $\begin{array}{c}11 \\
(100.0) \\
\end{array}$ \\
\hline \multirow{3}{*}{ 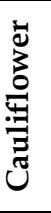 } & Marginal & 0 & 0 & 0 & 0 & $\begin{array}{c}0 \\
(0.0) \\
\end{array}$ & $\begin{array}{c}0 \\
(0.0) \\
\end{array}$ & $\begin{array}{c}0 \\
(0.0) \\
\end{array}$ & $\begin{array}{c}0 \\
(0.0) \\
\end{array}$ & $\begin{array}{c}0 \\
(0.0) \\
\end{array}$ & $\begin{array}{c}0 \\
(0.0) \\
\end{array}$ & $\begin{array}{c}0 \\
(0.0) \\
\end{array}$ & $\begin{array}{c}0 \\
(0.0) \\
\end{array}$ & $\begin{array}{c}0 \\
(0.0) \\
\end{array}$ & $\begin{array}{c}0 \\
(0.0) \\
\end{array}$ & $\begin{array}{c}0 \\
(0.0) \\
\end{array}$ & $\begin{array}{c}0 \\
(0.0) \\
\end{array}$ \\
\hline & Small & 565 & 0 & 0 & 565 & $\begin{array}{c}559 \\
(98.9) \\
\end{array}$ & $\begin{array}{c}0 \\
(0.0) \\
\end{array}$ & $\begin{array}{c}0 \\
(0.0) \\
\end{array}$ & $\begin{array}{c}559 \\
(98.9) \\
\end{array}$ & $\begin{array}{c}6 \\
(1.1) \\
\end{array}$ & $\begin{array}{c}0 \\
(0.0) \\
\end{array}$ & $\begin{array}{c}0 \\
(0.0) \\
\end{array}$ & $\begin{array}{c}6 \\
(1.1) \\
\end{array}$ & $\begin{array}{c}4 \\
(100.0) \\
\end{array}$ & $\begin{array}{c}0 \\
(0.0) \\
\end{array}$ & $\begin{array}{c}0 \\
(0.0) \\
\end{array}$ & $\begin{array}{c}4 \\
(100.0) \\
\end{array}$ \\
\hline & Large & 330 & 78 & 156 & 564 & $\begin{array}{c}305 \\
(92.4) \\
\end{array}$ & $\begin{array}{c}68 \\
(87.2) \\
\end{array}$ & $\begin{array}{c}152 \\
(97.4) \\
\end{array}$ & $\begin{array}{c}525 \\
(94.4) \\
\end{array}$ & $\begin{array}{c}25 \\
(7.6) \\
\end{array}$ & $\begin{array}{c}10 \\
(12.8) \\
\end{array}$ & $\begin{array}{c}4 \\
(2.6) \\
\end{array}$ & $\begin{array}{c}39 \\
(6.9) \\
\end{array}$ & $\begin{array}{c}3 \\
(100.0) \\
\end{array}$ & $\begin{array}{c}1 \\
(100.0) \\
\end{array}$ & $\begin{array}{c}2 \\
(100.0)\end{array}$ & $\begin{array}{c}6 \\
(100.0) \\
\end{array}$ \\
\hline
\end{tabular}




\begin{tabular}{|c|c|c|c|c|c|c|c|c|c|c|c|c|c|c|c|c|c|}
\hline \multirow{3}{*}{ 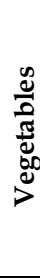 } & \multirow{3}{*}{ 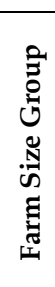 } & \multicolumn{4}{|c|}{$\mathrm{I}(\mathrm{P}-\mathrm{C})$} & \multicolumn{4}{|c|}{$\begin{array}{c}\text { No. of } \\
\text { Farmers }\end{array}$} & \multicolumn{4}{|c|}{$\begin{array}{c}\text { II ( } \\
\text { P-W-R-C) }\end{array}$} & \multicolumn{4}{|c|}{$\begin{array}{c}\text { No. of } \\
\text { Farmers }\end{array}$} \\
\hline & & $\begin{array}{l}\overrightarrow{0} \\
\frac{\pi}{\tilde{z}} \\
\frac{\pi}{2}\end{array}$ & 胥 & 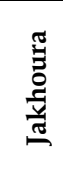 & 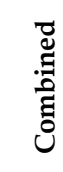 & 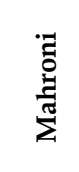 & 胥 & 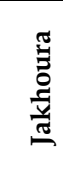 & 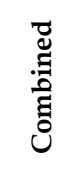 & $\begin{array}{l}\tilde{z} \\
\frac{\tilde{d}}{\pi} \\
\sum\end{array}$ & 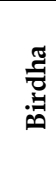 & 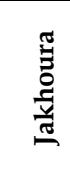 & 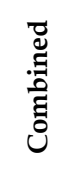 & 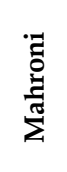 & 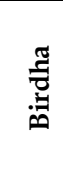 & 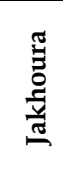 & 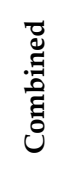 \\
\hline & & (17) & (18) & (19) & (20) & (21) & (22) & (23) & (24) & (25) & (26) & (27) & (28) & (29) & (30) & (31) & (32) \\
\hline \multirow{3}{*}{ 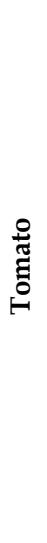 } & 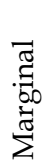 & 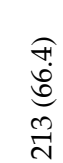 & 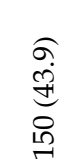 & 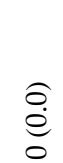 & 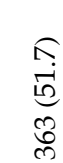 & 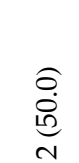 & 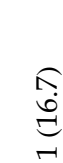 & $\begin{array}{l}\widehat{o} \\
\stackrel{\dot{e}}{0}\end{array}$ & 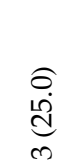 & $\begin{array}{l}0 \\
\dot{d} \\
\mathbb{C} \\
\infty \\
0\end{array}$ & $\begin{array}{l}\text { శ్ } \\
\text { ద్ర } \\
\text { హ̆ }\end{array}$ & 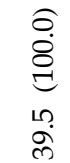 & 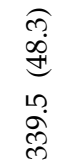 & $\begin{array}{l}\hat{\sigma} \\
\text { bi } \\
\text { b. }\end{array}$ & $\begin{array}{c}\widehat{m} \\
\hat{\infty} \\
\frac{D}{n}\end{array}$ & $\begin{array}{l}\hat{0} \\
\stackrel{0}{0} \\
\text { di }\end{array}$ & $\begin{array}{l}\widehat{\sigma} \\
\dot{1} \\
\alpha\end{array}$ \\
\hline & $\begin{array}{l}\text { ज్ } \\
\text { कี }\end{array}$ & 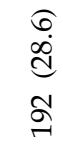 & $\begin{array}{l}\widehat{\Theta} \\
\stackrel{\dot{e}}{\circ}\end{array}$ & $\begin{array}{l}\overparen{f} \\
\stackrel{d}{d} \\
\text { s }\end{array}$ & 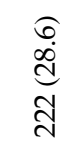 & 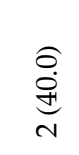 & 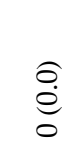 & 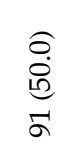 & $\begin{array}{l}\widehat{\cdots} \\
\stackrel{0}{0} \\
\infty\end{array}$ & 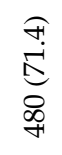 & 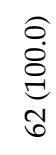 & $\begin{array}{l}\widehat{6} \\
\circ \\
\hat{E} \\
\hat{G}\end{array}$ & 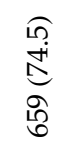 & $\begin{array}{l}\hat{0} \\
\stackrel{0}{0} \\
\text { co }\end{array}$ & $\begin{array}{l}\widehat{O} \\
\stackrel{8}{0} \\
\stackrel{C}{N}\end{array}$ & $\begin{array}{l}\hat{\theta} \\
\stackrel{0}{0} \\
\stackrel{0}{r}\end{array}$ & 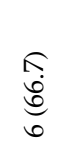 \\
\hline & 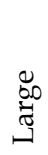 & 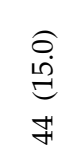 & $\begin{array}{l}\stackrel{\varrho}{\dot{e}} \\
\stackrel{0}{e}\end{array}$ & $\begin{array}{l}\hat{0} \\
\stackrel{e}{e} \\
0\end{array}$ & $\begin{array}{l}\text { In } \\
\text { İ } \\
\text { J }\end{array}$ & 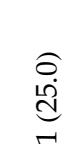 & $\stackrel{0}{0}$ & $\stackrel{0}{0}$ & $\underset{7}{-1}$ & 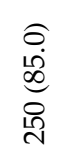 & 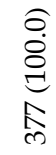 & $\begin{array}{l}\hat{n} \\
\hat{\alpha} \\
\vec{\sigma}\end{array}$ & $\begin{array}{l}\hat{\sigma} \\
\mathscr{\Omega} \\
\infty \\
\infty \\
\delta\end{array}$ & 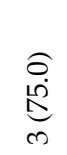 & $\underset{\infty}{\stackrel{0}{\stackrel{0}{0}}}$ & 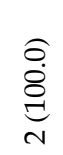 & $\begin{array}{l}\widehat{\hat{i}} \\
\text { } \\
\stackrel{\sigma}{2}\end{array}$ \\
\hline
\end{tabular}




\begin{tabular}{|c|c|c|c|c|c|c|c|c|c|c|c|c|c|c|c|c|c|}
\hline \multirow{3}{*}{ 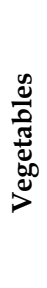 } & \multirow{3}{*}{ 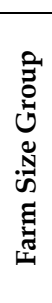 } & \multicolumn{4}{|c|}{$\mathrm{I}(\mathrm{P}-\mathrm{C})$} & \multicolumn{4}{|c|}{$\begin{array}{c}\text { No. of } \\
\text { Farmers }\end{array}$} & \multicolumn{4}{|c|}{$\begin{array}{c}\text { II } \\
\text { (P-W-R-C) } \\
\end{array}$} & \multicolumn{4}{|c|}{$\begin{array}{c}\text { No. of } \\
\text { Farmers }\end{array}$} \\
\hline & & 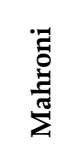 & 泀 & 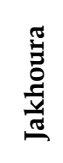 & 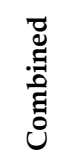 & $\begin{array}{l}\tilde{D} \\
\frac{\tilde{d}}{\pi} \\
\sum\end{array}$ & 苞 & 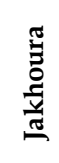 & 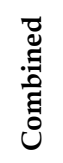 & 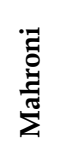 & 泀 & 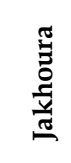 & 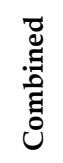 & $\begin{array}{l}\tilde{D} \\
\frac{\pi}{\pi} \\
\Sigma\end{array}$ & 泀 & 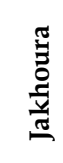 & 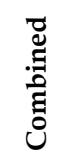 \\
\hline & & (17) & (18) & (19) & (20) & (21) & (22) & (23) & (24) & (25) & (26) & (27) & (28) & (29) & (30) & (31) & (32) \\
\hline \multirow{3}{*}{ 苞 } & 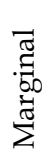 & 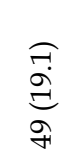 & $\begin{array}{l}\widehat{\infty} \\
\tilde{c} \\
\tilde{c} \\
\tilde{A}\end{array}$ & $\underset{0}{\stackrel{0}{0}}$ & 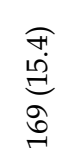 & $\underset{\sim}{\stackrel{d}{d}}$ & $\begin{array}{c}\widehat{\infty} \\
\stackrel{0}{0} \\
\stackrel{0}{0}\end{array}$ & $\stackrel{0}{\stackrel{0}{0}}$ & $\begin{array}{l}\text { d. } \\
\underset{d}{d}\end{array}$ & $\begin{array}{l}\widehat{\sigma} \\
\dot{d} \\
\infty \\
\stackrel{d}{d}\end{array}$ & 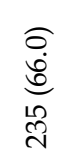 & 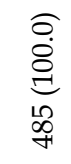 & 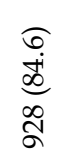 & $\begin{array}{l}\stackrel{\partial}{\dot{\Phi}} \\
\stackrel{\infty}{+}\end{array}$ & $\begin{array}{l}\underset{\hat{\sigma}}{\delta} \\
\underset{f}{+}\end{array}$ & 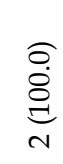 & $\begin{array}{l}\sigma \\
\delta \\
\delta \\
\circ\end{array}$ \\
\hline & $\begin{array}{l}\text { ₹ี } \\
\text { कี }\end{array}$ & $\begin{array}{l}\text { of } \\
\text { ti } \\
\text { 용 }\end{array}$ & $\underset{\stackrel{o}{\ominus}}{\stackrel{0}{0}}$ & 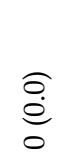 & 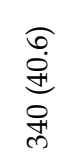 & $\underset{\sim}{\stackrel{d}{d}}$ & 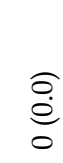 & 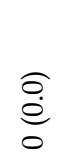 & $\stackrel{\overparen{9}}{\overparen{a}}$ & $\begin{array}{l}\text { f } \\
\ddot{0} \\
0 \\
\infty \\
\infty\end{array}$ & 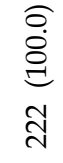 & 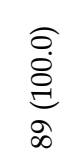 & 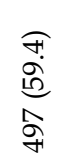 & 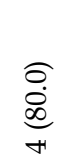 & $\begin{array}{l}\stackrel{0}{0} \\
\stackrel{8}{0} \\
\stackrel{d}{d}\end{array}$ & 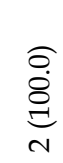 & $\begin{array}{l}\widehat{\sigma} \\
\stackrel{8}{\circ} \\
\stackrel{1}{0}\end{array}$ \\
\hline & 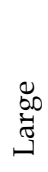 & 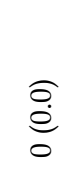 & $\underset{\stackrel{o}{e}}{\stackrel{0}{0}}$ & $\underset{0}{\stackrel{0}{0}}$ & $\underset{\stackrel{o}{e}}{\stackrel{0}{0}}$ & $\underset{\stackrel{o}{e}}{\stackrel{0}{0}}$ & $\stackrel{0}{\stackrel{0}{e}}$ & $\stackrel{0}{\stackrel{0}{0}}$ & 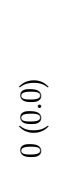 & 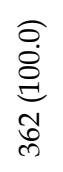 & 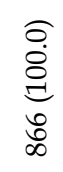 & 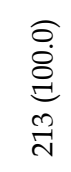 & 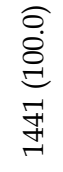 & $\begin{array}{l}\stackrel{\sigma}{\dot{Q}} \\
\stackrel{0}{0} \\
\infty\end{array}$ & $\begin{array}{l}\stackrel{\Xi}{\dot{\theta}} \\
\stackrel{8}{E}\end{array}$ & 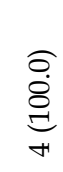 & 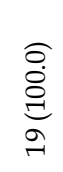 \\
\hline
\end{tabular}




\begin{tabular}{|c|c|c|c|c|c|c|c|c|c|c|c|c|c|c|c|c|c|}
\hline \multirow{3}{*}{ 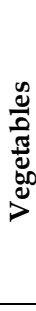 } & \multirow{3}{*}{ 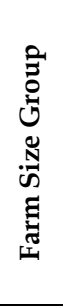 } & \multicolumn{4}{|c|}{$\mathrm{I}(\mathrm{P}-\mathrm{C})$} & \multicolumn{4}{|c|}{$\begin{array}{l}\text { No. of } \\
\text { Farmers }\end{array}$} & \multicolumn{4}{|c|}{$\begin{array}{c}\text { II } \\
\text { (P-W-R-C) }\end{array}$} & \multicolumn{4}{|c|}{$\begin{array}{l}\text { No. of } \\
\text { Farmers }\end{array}$} \\
\hline & & $\begin{array}{l}\tilde{D} \\
\frac{0}{\pi} \\
\sum\end{array}$ & 苛 & 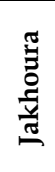 & 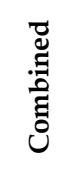 & 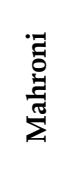 & 节 & 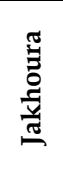 & 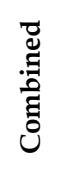 & 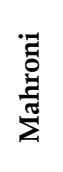 & 胥 & 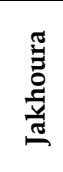 & 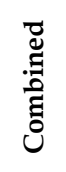 & 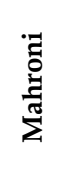 & 苟 & 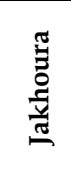 & 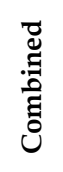 \\
\hline & & (17) & $(18)$ & (19) & $(20)$ & (21) & (22) & (23) & (24) & (25) & (26) & (27) & $(28)$ & (29) & (30) & $(31)$ & (32) \\
\hline \multirow{3}{*}{ 总 } & 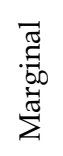 & $\begin{array}{l}\overparen{F} \\
\stackrel{+}{+} \\
\text { స్ }\end{array}$ & $\begin{array}{l}0 \\
\text { id } \\
\stackrel{d}{0} \\
\end{array}$ & $\stackrel{\overparen{\varrho}}{\stackrel{0}{0}}$ & 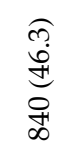 & 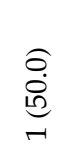 & 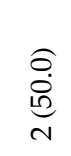 & $\begin{array}{l}\stackrel{0}{0} \\
\stackrel{0}{0}\end{array}$ & $\begin{array}{l}\widehat{\widetilde{D}} \\
\stackrel{0}{\tilde{D}}\end{array}$ & $\begin{array}{l}0 \\
\stackrel{0}{0} \\
\stackrel{0}{0} \\
\stackrel{\sim}{N}\end{array}$ & 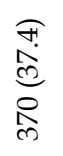 & $\begin{array}{l}\stackrel{0}{0} \\
\stackrel{0}{0} \\
\stackrel{0}{0} \\
\end{array}$ & 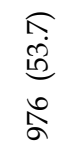 & $\begin{array}{l}\underset{\dot{\theta}}{0} \\
\underset{n}{0}\end{array}$ & 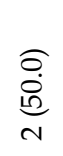 & $\underset{0}{\stackrel{0}{0}}$ & $\begin{array}{l}\hat{R} \\
\text { dig } \\
\sigma\end{array}$ \\
\hline & $\begin{array}{l}\text { Еี } \\
\text { का }\end{array}$ & $\begin{array}{l}\widehat{\alpha} \\
\infty \\
\infty \\
0 \\
0 \\
\stackrel{f}{\infty}\end{array}$ & $\begin{array}{l}\hat{\varrho} \\
\stackrel{0}{0}\end{array}$ & $\stackrel{\varrho}{\stackrel{\varrho}{0}}$ & 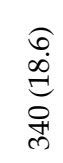 & 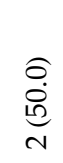 & $\begin{array}{l}\hat{O} \\
\stackrel{0}{0}\end{array}$ & $\stackrel{\varrho}{\stackrel{\leftrightarrow}{0}}$ & $\begin{array}{l}\widehat{a} \\
\stackrel{d}{d} \\
\stackrel{d}{d}\end{array}$ & 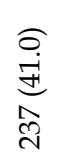 & 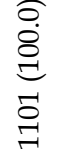 & 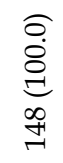 & $\begin{array}{l}\stackrel{\Im}{+} \\
\stackrel{+\infty}{\infty} \\
\stackrel{\infty}{+} \\
\stackrel{+}{+}\end{array}$ & $\begin{array}{l}\widehat{\partial} \\
\stackrel{b}{0} \\
\text { ven }\end{array}$ & 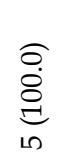 & $\begin{array}{l}\hat{O} \\
\stackrel{0}{0} \\
\stackrel{0}{a}\end{array}$ & $\begin{array}{l}\widehat{\widehat{o}} \\
\stackrel{\Phi}{\infty} \\
\sigma\end{array}$ \\
\hline & 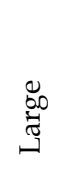 & $\begin{array}{l}\hat{\sigma} \\
\dot{d} \\
\text { d } \\
\text { के }\end{array}$ & $\begin{array}{l}\hat{\varrho} \\
\dot{e} \\
0\end{array}$ & $\stackrel{\circ}{\dot{q}}$ & ڤั & 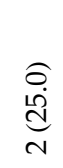 & $\begin{array}{l}\hat{\varrho} \\
\stackrel{\leftrightarrow}{0}\end{array}$ & $\stackrel{\varrho}{\stackrel{\leftrightarrow}{0}}$ & గก & 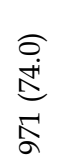 & ठิ. & $\begin{array}{l}\widehat{o} \\
\stackrel{0}{0} \\
o \\
\not{q}\end{array}$ & 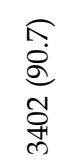 & 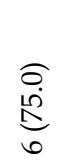 & 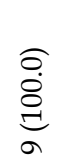 & $\begin{array}{l}\widehat{O} \\
\stackrel{8}{0} \\
\stackrel{0}{+}\end{array}$ & $\begin{array}{l}\text { గn } \\
0 \\
0 \\
\sigma \\
\sigma\end{array}$ \\
\hline
\end{tabular}




\begin{tabular}{|c|c|c|c|c|c|c|c|c|c|c|c|c|c|c|c|c|c|}
\hline \multirow{3}{*}{ 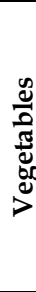 } & \multirow{3}{*}{ 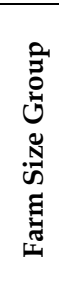 } & \multicolumn{4}{|c|}{$\mathrm{I}(\mathrm{P}-\mathrm{C})$} & \multicolumn{4}{|c|}{$\begin{array}{c}\text { No. of } \\
\text { Farmers }\end{array}$} & \multicolumn{4}{|c|}{$\begin{array}{c}\text { II } \\
\text { (P-W-R-C) }\end{array}$} & \multicolumn{4}{|c|}{$\begin{array}{l}\text { No. of } \\
\text { Farmers }\end{array}$} \\
\hline & & 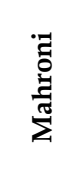 & 䞤 & 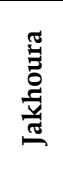 & 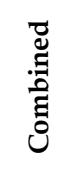 & $\begin{array}{l}\tilde{D} \\
\stackrel{\Xi}{\pi} \\
\sum\end{array}$ & 䞤 & 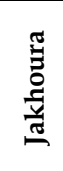 & 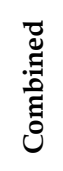 & 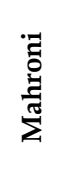 & 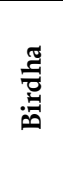 & 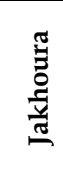 & $\begin{array}{l}\widetilde{\Xi} \\
\text { : } \\
\overline{0} \\
\dot{0}\end{array}$ & $\begin{array}{l}\tilde{z} \\
\bar{z} \\
\bar{z}\end{array}$ & 荡 & 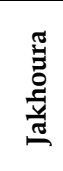 & 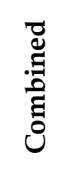 \\
\hline & & $\begin{array}{l}\text { (17) } \\
\text { (a) }\end{array}$ & \begin{tabular}{|l|} 
(18) \\
\end{tabular} & (19) & (20) & (21) & (22) & (23) & (24) & (25) & (26) & $\begin{array}{l}\text { (27) } \\
\end{array}$ & (28) & $\begin{array}{l}\text { (29) } \\
\end{array}$ & $\begin{array}{l}(30) \\
\end{array}$ & $\begin{array}{l}\text { (31) } \\
\text { (3) }\end{array}$ & (32) \\
\hline \multirow{3}{*}{ 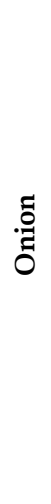 } & 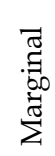 & 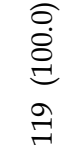 & 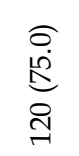 & $\stackrel{a}{\stackrel{0}{0}}$ & $\begin{array}{l}\sigma \\
\hat{1} \\
\text { d. } \\
\text { ते }\end{array}$ & $\begin{array}{l}\underset{0}{\dot{g}} \\
\underset{0}{0}\end{array}$ & $\begin{array}{l}\text { ल. } \\
\text { लें } \\
\text { तै }\end{array}$ & $\stackrel{0}{0}$ & 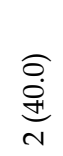 & $\underset{0}{\stackrel{0}{e}}$ & $\begin{array}{l}\text { ô } \\
\text { d.d } \\
\text { o }\end{array}$ & $\begin{array}{l}\widehat{O} \\
\stackrel{8}{0} \\
\stackrel{0}{0} \\
\text { iे }\end{array}$ & $\begin{array}{l}\stackrel{\text { f }}{d} \\
\text { a }\end{array}$ & $\stackrel{\varrho}{\stackrel{0}{0}}$ & 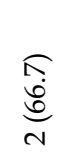 & 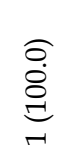 & $\begin{array}{l}\widehat{o} \\
\stackrel{0}{0} \\
\stackrel{0}{m}\end{array}$ \\
\hline & $\begin{array}{l}\text { च } \\
\text { है }\end{array}$ & 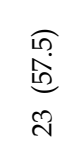 & $\begin{array}{l}\hat{o} \\
\stackrel{0}{0} \\
\stackrel{0}{o}\end{array}$ & $\begin{array}{l}\hat{\stackrel{a}{0}} \\
\stackrel{0}{0}\end{array}$ & 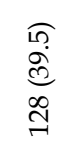 & 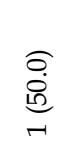 & $\begin{array}{l}\hat{0} \\
\stackrel{\dot{\theta}}{0}\end{array}$ & 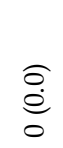 & 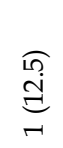 & 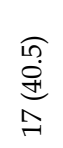 & $\begin{array}{l}\stackrel{a}{\dot{\theta}} \\
\stackrel{0}{0} \\
\stackrel{\leftrightarrow}{\circ}\end{array}$ & 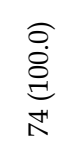 & $\begin{array}{l}\tilde{1} \\
0 \\
0 \\
0 \\
\circ \\
\sigma\end{array}$ & $\begin{array}{l}\widehat{\partial} \\
\stackrel{0}{0} \\
\underline{n}\end{array}$ & 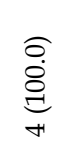 & 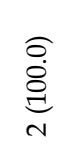 & 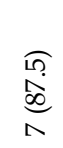 \\
\hline & $\begin{array}{l}\text {. } \\
\text { పే }\end{array}$ & 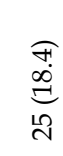 & 宊 & $\stackrel{\varrho}{\stackrel{0}{0}}$ & 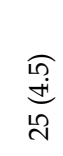 & 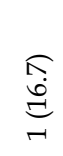 & 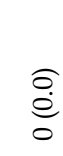 & $\stackrel{\widehat{\grave{\theta}}}{\stackrel{0}{0}}$ & $\underset{\sigma}{\underset{\sigma}{\sigma}}$ & $\begin{array}{l}\sigma \\
\vec{b} \\
\vec{\infty} \\
\vec{\exists}\end{array}$ & $\begin{array}{l}\stackrel{0}{0} \\
\stackrel{0}{0} \\
\stackrel{0}{\circ} \\
\stackrel{\infty}{\sim}\end{array}$ & $\begin{array}{l}\widehat{O} \\
\stackrel{0}{0} \\
\stackrel{0}{0} \\
\tilde{\sim}\end{array}$ & 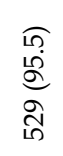 & $\begin{array}{c}\widehat{\widetilde{0}} \\
\stackrel{\infty}{\infty} \\
\frac{\infty}{n}\end{array}$ & $\begin{array}{l}\stackrel{0}{0} \\
\stackrel{\vec{\theta}}{0}\end{array}$ & 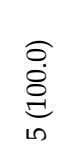 & $\begin{array}{l}\widehat{F} \\
\stackrel{\vec{d}}{\sigma}\end{array}$ \\
\hline
\end{tabular}




\begin{tabular}{|c|c|c|c|c|c|c|c|c|c|c|c|c|c|c|c|c|c|}
\hline \multirow{3}{*}{ 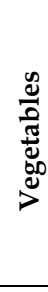 } & \multirow{3}{*}{ 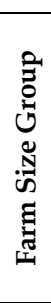 } & \multicolumn{4}{|c|}{$\mathrm{I}(\mathrm{P}-\mathrm{C})$} & \multicolumn{4}{|c|}{$\begin{array}{c}\text { No. of } \\
\text { Farmers }\end{array}$} & \multicolumn{4}{|c|}{$\begin{array}{c}\text { II } \\
\text { (P-W-R-C) }\end{array}$} & \multicolumn{4}{|c|}{$\begin{array}{c}\text { No. of } \\
\text { Farmers }\end{array}$} \\
\hline & & 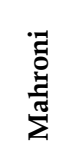 & 窇 & 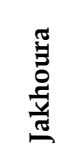 & 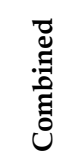 & 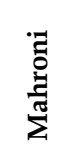 & 苞 & 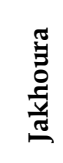 & 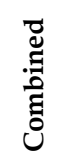 & 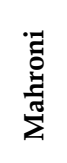 & 吾 & 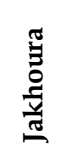 & $\begin{array}{l}\widetilde{\mathscr{U}} \\
\stackrel{.0}{0} \\
\bar{\Xi} \\
0\end{array}$ & 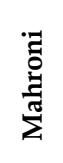 & 吾 & 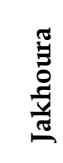 & 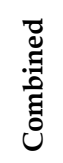 \\
\hline & & $\begin{array}{l}\text { (17) } \\
\text { (a) }\end{array}$ & $\begin{array}{l}\text { (18) } \\
\end{array}$ & (19) & (20) & $\begin{array}{l}(21) \\
\end{array}$ & (22) & (23) & $\begin{array}{l}\text { (24) } \\
\end{array}$ & (25) & $\begin{array}{l}\text { (26) } \\
\end{array}$ & $\begin{array}{l}\text { (27) } \\
\end{array}$ & (28) & $\begin{array}{l}(29) \\
\end{array}$ & (30) & $\begin{array}{l}(31) \\
\end{array}$ & $\begin{array}{l}\text { (32) } \\
\end{array}$ \\
\hline \multirow{3}{*}{$\frac{\Xi}{0}$} & 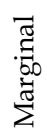 & $\begin{array}{l}0 \\
\stackrel{8}{0} \\
\text { o } \\
\stackrel{g}{\sigma}\end{array}$ & 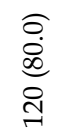 & 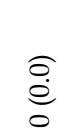 & $\begin{array}{l}\widehat{a} \\
\text { है } \\
\text { ते }\end{array}$ & 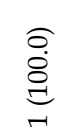 & 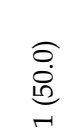 & $\begin{array}{l}\hat{\dot{\theta}} \\
\stackrel{0}{0}\end{array}$ & $\begin{array}{l}\widehat{a} \\
\text { bi } \\
\text { N. }\end{array}$ & $\begin{array}{l}\hat{O} \\
\dot{e} \\
0\end{array}$ & $\begin{array}{l}\text { ồ. } \\
\text { d. } \\
\text { o }\end{array}$ & $\begin{array}{l}\hat{a} \\
\stackrel{0}{0} \\
\stackrel{0}{a} \\
\text { o. }\end{array}$ & $\begin{array}{l}\text { d } \\
\text { d } \\
\text { a }\end{array}$ & $\begin{array}{l}\hat{\stackrel{\theta}{0}} \\
\stackrel{0}{0}\end{array}$ & 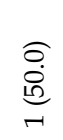 & $\underset{-}{\stackrel{0}{\stackrel{0}{0}}}$ & 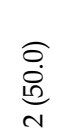 \\
\hline & 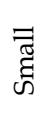 & $\begin{array}{l}\sigma \\
\underset{+}{+} \\
\infty \\
\infty\end{array}$ & $\underset{\stackrel{0}{0}}{\stackrel{0}{0}}$ & 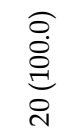 & $\begin{array}{l}10 \\
0 \\
0 \\
0 \\
0 \\
0\end{array}$ & 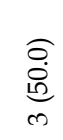 & 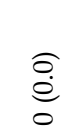 & 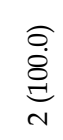 & 命 & $\begin{array}{l}\text { 㝵 } \\
\text { 总 } \\
\text { o }\end{array}$ & 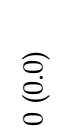 & 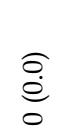 & 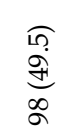 & $\begin{array}{l}\widehat{a} \\
\text { in } \\
\text { con }\end{array}$ & 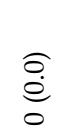 & $\stackrel{\widehat{\dot{\theta}}}{0}$ & $\begin{array}{l}\frac{1}{1} \\
0 \\
0 \\
0\end{array}$ \\
\hline & $\begin{array}{l}\text { 品 } \\
\text { 亗 }\end{array}$ & $\begin{array}{l}\hat{\sigma} \\
\hat{\sigma} \\
\stackrel{\infty}{\infty}\end{array}$ & $\begin{array}{l}\hat{\stackrel{\theta}{0}} \\
\stackrel{0}{0}\end{array}$ & $\begin{array}{l}\hat{\dot{\theta}} \\
\stackrel{0}{0}\end{array}$ & $\begin{array}{l}\widehat{T} \\
\stackrel{0}{0} \\
\stackrel{\infty}{\infty}\end{array}$ & 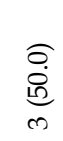 & $\begin{array}{l}\widehat{o} \\
\stackrel{\dot{\theta}}{0}\end{array}$ & $\begin{array}{l}\stackrel{a}{\dot{\theta}} \\
\stackrel{0}{0}\end{array}$ & $\begin{array}{l}\widehat{m} \\
\text { dn } \\
\text { लn }\end{array}$ & 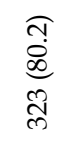 & 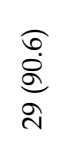 & $\begin{array}{l}\hat{a} \\
\stackrel{0}{0} \\
\stackrel{0}{\circ} \\
\&\end{array}$ & 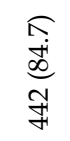 & 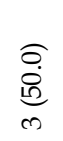 & $\underset{-\sigma}{\stackrel{\sigma}{0}}$ & 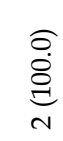 & 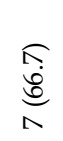 \\
\hline
\end{tabular}




\begin{tabular}{|c|c|c|c|c|c|c|c|c|c|c|c|c|c|c|c|c|c|}
\hline \multirow{3}{*}{ 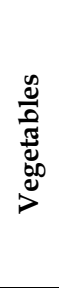 } & \multirow{3}{*}{ 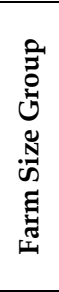 } & \multicolumn{4}{|c|}{$\mathrm{I}(\mathrm{P}-\mathrm{C})$} & \multicolumn{4}{|c|}{$\begin{array}{c}\text { No. of } \\
\text { Farmers }\end{array}$} & \multicolumn{4}{|c|}{$\begin{array}{c}\text { II } \\
\text { (P-W-R-C) }\end{array}$} & \multicolumn{4}{|c|}{$\begin{array}{c}\text { No. of } \\
\text { Farmers }\end{array}$} \\
\hline & & 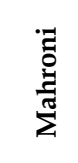 & 苛 & 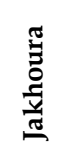 & 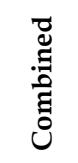 & 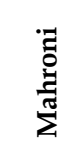 & 胥 & 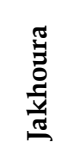 & 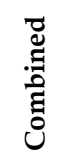 & $\begin{array}{l}\tilde{d} \\
\bar{c} \\
\tilde{\Sigma}\end{array}$ & 胥 & 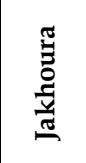 & 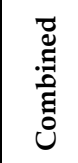 & $\begin{array}{l}\tilde{D} \\
\frac{\pi}{\pi} \\
\Sigma\end{array}$ & 苛 & 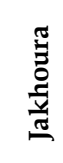 & 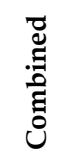 \\
\hline & & (17) & (18) & (19) & (20) & (21) & (22) & (23) & (24) & (25) & \begin{tabular}{|l|} 
(26) \\
\end{tabular} & \begin{tabular}{|l|}
$(27)$ \\
\end{tabular} & \begin{tabular}{|l|}
$(28)$ \\
\end{tabular} & (29) & (30) & (31) & (32) \\
\hline \multirow{3}{*}{$\begin{array}{l}\bar{\Xi} \\
0 \\
0 \\
0 \\
\bar{E} \\
0\end{array}$} & 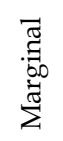 & 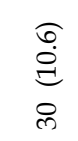 & 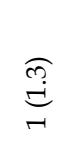 & $\begin{array}{l}\hat{0} \\
\stackrel{e}{0}\end{array}$ & 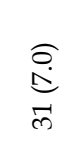 & 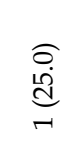 & 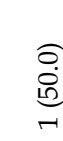 & 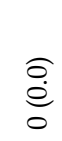 & $\begin{array}{l}\widehat{\sigma} \\
\stackrel{d}{d} \\
\stackrel{d}{d}\end{array}$ & 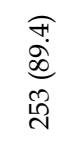 & $\begin{array}{l}\widehat{\widehat{\alpha}} \\
\infty \\
0 \\
\infty \\
\infty \\
\infty\end{array}$ & $\begin{array}{l}\stackrel{0}{0} \\
\stackrel{0}{0} \\
\stackrel{\infty}{\infty}\end{array}$ & $\begin{array}{l}\widehat{\sigma} \\
\infty \\
\sigma \\
\vec{y}\end{array}$ & 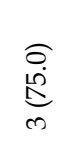 & 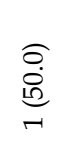 & 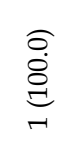 & $\underset{n}{\stackrel{f}{5}}$ \\
\hline & $\begin{array}{l}\overline{\widetilde{\widetilde{J}}} \\
\text { की }\end{array}$ & สิ & 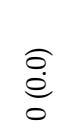 & $\begin{array}{l}\widehat{\alpha} \\
\stackrel{\infty}{d} \\
\stackrel{\infty}{\infty}\end{array}$ & 号 & $\underset{r}{\stackrel{\widehat{d}}{a}}$ & $\stackrel{\partial}{\stackrel{0}{e}}$ & 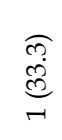 & $\widehat{\widetilde{d}}$ & $\stackrel{\overbrace{}}{\stackrel{\overbrace{}}{E}}$ & Fُ & 莺牻 & 范 & $\underset{\mathrm{c}}{\stackrel{\mathrm{p}}{\mathrm{s}}}$ & 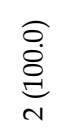 & 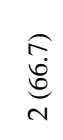 & 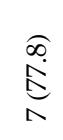 \\
\hline & $\begin{array}{l}\text { 品 } \\
\text { పే }\end{array}$ & $\begin{array}{l}\widehat{\widetilde{m}} \\
\overrightarrow{\mathrm{d}} \\
\stackrel{\text { సे }}{7}\end{array}$ & $\underset{\stackrel{o}{\dot{e}}}{\stackrel{0}{0}}$ & $\stackrel{\hat{o}}{\stackrel{0}{0}}$ & 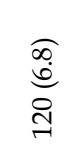 & 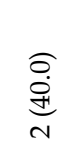 & อ̊ं & 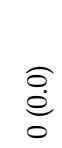 & 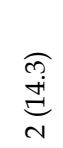 & $\begin{array}{l}0 \\
0 \\
0 \\
0 \\
\mathbb{0} \\
\text { d }\end{array}$ & $\begin{array}{l}\text { o. } \\
\dot{0} \\
\text { d् } \\
\text { fid }\end{array}$ & 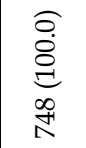 & 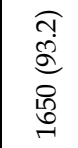 & $\begin{array}{l}\hat{\sigma} \\
\stackrel{0}{0} \\
\frac{0}{m}\end{array}$ & 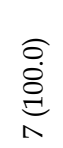 & 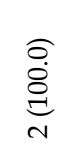 & 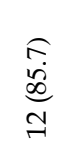 \\
\hline
\end{tabular}




\begin{tabular}{|c|c|c|c|c|c|c|c|c|c|c|c|c|c|c|c|c|c|}
\hline \multirow{3}{*}{ 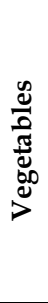 } & \multirow{3}{*}{ 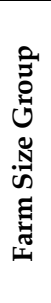 } & \multicolumn{4}{|c|}{$\mathrm{I}(\mathrm{P}-\mathrm{C})$} & \multicolumn{4}{|c|}{$\begin{array}{l}\text { No. of } \\
\text { Farmers }\end{array}$} & \multicolumn{4}{|c|}{$\begin{array}{c}\text { II } \\
\text { (P-W-R-C) }\end{array}$} & \multicolumn{4}{|c|}{$\begin{array}{l}\text { No. of } \\
\text { Farmers }\end{array}$} \\
\hline & & 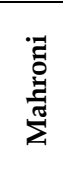 & 荡 & 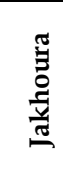 & 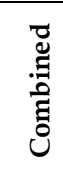 & $\begin{array}{l}\tilde{\Xi} \\
\frac{\tilde{g}}{\pi} \\
\sum\end{array}$ & 苛 & 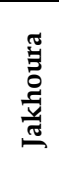 & 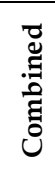 & 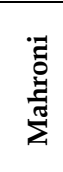 & 胥 & 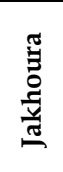 & 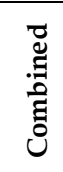 & $\begin{array}{l}\ddot{\Xi} \\
\bar{d} \\
\bar{\pi}\end{array}$ & 胥 & $\begin{array}{l}\widetilde{5} \\
\text { है } \\
\text { 竘 }\end{array}$ & 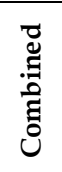 \\
\hline & & (17) & (18) & (19) & (20) & (21) & (22) & (23) & (24) & (25) & (26) & (27) & (28) & (29) & (30) & (31) & (32) \\
\hline \multirow{3}{*}{ 莕 } & 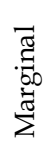 & $\begin{array}{l}\stackrel{\widehat{o}}{\dot{e}} \\
\text { and }\end{array}$ & $\begin{array}{l}\widehat{O} \\
\stackrel{0}{0}\end{array}$ & $\begin{array}{l}\widehat{o} \\
\stackrel{\dot{\theta}}{0}\end{array}$ & $\begin{array}{l}\widehat{o} \\
\stackrel{\dot{\theta}}{0}\end{array}$ & 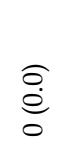 & $\begin{array}{l}\widehat{c} \\
\stackrel{\dot{\theta}}{0}\end{array}$ & $\begin{array}{l}\hat{e} \\
\stackrel{\dot{\theta}}{0}\end{array}$ & $\begin{array}{l}\hat{e} \\
\stackrel{\dot{\theta}}{0}\end{array}$ & 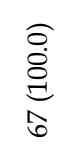 & 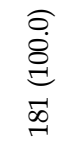 & $\begin{array}{l}\text { o̊ } \\
\stackrel{0}{0} \\
\stackrel{0}{\sigma}\end{array}$ & $\begin{array}{l}\widehat{a} \\
\stackrel{0}{0} \\
\text { à } \\
\text { à }\end{array}$ & $\begin{array}{l}\stackrel{0}{0} \\
\stackrel{8}{0} \\
\underset{-}{0}\end{array}$ & 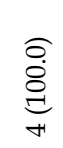 & $\begin{array}{l}\underset{\dot{\theta}}{0} \\
\underset{-}{0}\end{array}$ & $\begin{array}{l}\text { } \\
\stackrel{0}{0} \\
\stackrel{0}{0}\end{array}$ \\
\hline & 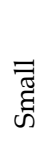 & 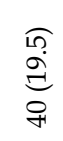 & 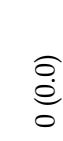 & $\begin{array}{l}\stackrel{\varrho}{0} \\
\stackrel{0}{0}\end{array}$ & 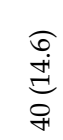 & $\begin{array}{l}\widehat{m} \\
\hat{d} \\
\vec{c}\end{array}$ & $\begin{array}{l}\widehat{o} \\
\stackrel{\dot{e}}{0}\end{array}$ & 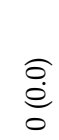 & $\begin{array}{l}\stackrel{0}{d} \\
\stackrel{d}{d}\end{array}$ & 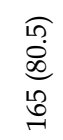 & $\begin{array}{l}\widehat{O} \\
\stackrel{0}{0} \\
\stackrel{0}{0} \\
R\end{array}$ & $\begin{array}{l}\widehat{\grave{\theta}} \\
\stackrel{\dot{0}}{\circ}\end{array}$ & 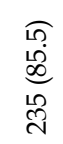 & $\begin{array}{l}\hat{R} \\
\delta \\
\delta \\
\delta\end{array}$ & 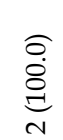 & 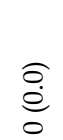 & $\begin{array}{l}\hat{\mathscr{O}} \\
\stackrel{\dot{D}}{\vec{H}}\end{array}$ \\
\hline & 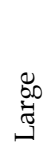 & $\begin{array}{l}\sigma \\
\dot{0} \\
\stackrel{0}{0} \\
\swarrow 2\end{array}$ & $\begin{array}{l}\stackrel{0}{0} \\
\stackrel{0}{0}\end{array}$ & $\begin{array}{l}\stackrel{\varrho}{0} \\
\stackrel{0}{0}\end{array}$ & $\begin{array}{l}\text { तू } \\
\text { dે } \\
\text { ूू }\end{array}$ & 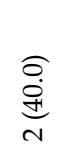 & $\begin{array}{l}\stackrel{\varrho}{0} \\
\stackrel{0}{0}\end{array}$ & $\begin{array}{l}\stackrel{\varrho}{0} \\
\stackrel{0}{0}\end{array}$ & 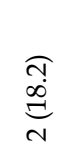 & $\begin{array}{l}\overparen{\not} \\
\text { d్ } \\
\text { ते }\end{array}$ & 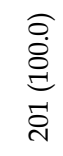 & $\begin{array}{l}\hat{O} \\
\stackrel{0}{0}\end{array}$ & 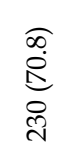 & $\begin{array}{l}\hat{0} \\
\stackrel{0}{0} \\
\text { m. }\end{array}$ & $\begin{array}{l}\widehat{O} \\
\stackrel{0}{0} \\
\stackrel{0}{0}\end{array}$ & $\stackrel{a}{\stackrel{0}{0}}$ & $\begin{array}{l}\widehat{\infty} \\
\vec{\infty} \\
\stackrel{\infty}{a}\end{array}$ \\
\hline
\end{tabular}




\begin{tabular}{|c|c|c|c|c|c|c|c|c|c|c|c|c|c|c|c|c|c|}
\hline \multirow{3}{*}{$\begin{array}{l}\frac{0}{0} \\
\frac{0}{0} \\
\frac{\pi}{4} \\
\frac{8}{2}\end{array}$} & \multirow{3}{*}{ 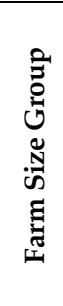 } & \multicolumn{4}{|c|}{ I (P-C) } & \multicolumn{4}{|c|}{$\begin{array}{c}\text { No. of } \\
\text { Farmers }\end{array}$} & \multicolumn{4}{|c|}{$\begin{array}{c}\text { II } \\
(\mathrm{P}-\mathrm{W}-\mathrm{R}-\mathrm{C})\end{array}$} & \multicolumn{4}{|c|}{$\begin{array}{c}\text { No. of } \\
\text { Farmers }\end{array}$} \\
\hline & & $\frac{\ddot{\Xi}}{\Xi^{J}}$ & 䒕 & 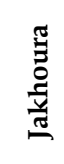 & 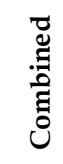 & 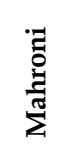 & 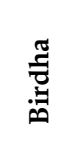 & 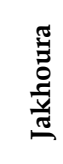 & 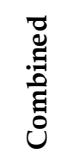 & $\begin{array}{l}\ddot{\Xi} \\
\frac{0}{\tilde{Z}} \\
\sum_{\Sigma}^{\pi}\end{array}$ & 疋 & 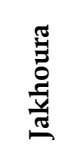 & 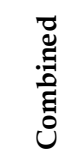 & $\begin{array}{l}\vec{\Xi} \\
\frac{0}{3} \\
\sum\end{array}$ & 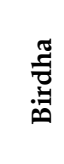 & 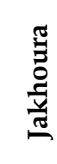 & 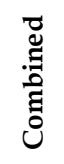 \\
\hline & & (17) & (18) & (19) & (20) & (21) & (22) & (23) & (24) & (25) & (26) & (27) & (28) & (29) & (30) & (31) & (32) \\
\hline \multirow{3}{*}{ 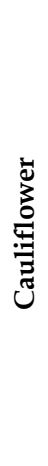 } & 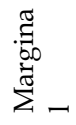 & $\underset{0}{\stackrel{0}{0}}$ & $\stackrel{0}{\stackrel{0}{0}}$ & $\underset{0}{\stackrel{0}{e}}$ & $\begin{array}{l}\stackrel{0}{0} \\
\stackrel{0}{0}\end{array}$ & $\begin{array}{l}\stackrel{0}{0} \\
\stackrel{e}{0}\end{array}$ & $\begin{array}{l}\stackrel{0}{0} \\
\stackrel{0}{0}\end{array}$ & $\underset{0}{\stackrel{0}{e}}$ & $\underset{0}{\stackrel{0}{e}}$ & $\begin{array}{l}\stackrel{0}{0} \\
\stackrel{0}{0}\end{array}$ & $\begin{array}{l}\partial \\
\stackrel{0}{0} \\
0\end{array}$ & $\underset{0}{\stackrel{0}{0}}$ & $\begin{array}{l}\stackrel{0}{0} \\
\stackrel{0}{0}\end{array}$ & $\underset{0}{\stackrel{0}{0}}$ & 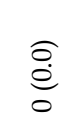 & $\begin{array}{l}\text { Oे } \\
\stackrel{e}{0} \\
0\end{array}$ & $\stackrel{0}{\stackrel{e}{0}}$ \\
\hline & 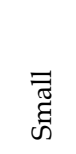 & 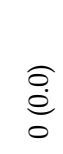 & $\underset{0}{\stackrel{0}{0}}$ & $\underset{0}{\stackrel{0}{e}}$ & $\underset{0}{\stackrel{0}{e}}$ & $\underset{0}{\stackrel{\partial}{e}}$ & $\underset{0}{\stackrel{0}{e}}$ & $\underset{0}{\stackrel{0}{e}}$ & $\underset{0}{\stackrel{\sigma}{e}}$ & 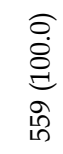 & $\begin{array}{l}0 \\
\stackrel{0}{0} \\
0\end{array}$ & $\underset{0}{\stackrel{\sigma}{0}}$ & $\begin{array}{l}\text { ठ̊. } \\
\stackrel{8}{0} \\
\stackrel{0}{0} \\
\text { مे }\end{array}$ & $\underset{\forall}{\stackrel{\partial}{\stackrel{\theta}{d}}}$ & $\underset{0}{\stackrel{0}{0}}$ & $\underset{0}{\stackrel{0}{0}}$ & 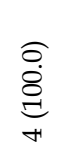 \\
\hline & 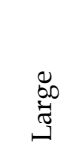 & $\begin{array}{l}\stackrel{0}{0} \\
\stackrel{0}{0}\end{array}$ & 兽 & 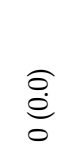 & 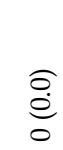 & $\begin{array}{l}a \\
\stackrel{0}{0} \\
0\end{array}$ & $\stackrel{a}{\stackrel{0}{e}}$ & 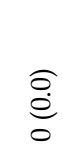 & $\begin{array}{l}a \\
\stackrel{0}{0} \\
0\end{array}$ & 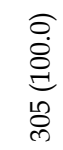 & $\begin{array}{l}\stackrel{0}{0} \\
\stackrel{0}{0} \\
\infty \\
0\end{array}$ & 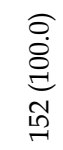 & 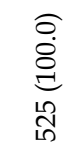 & $\underset{0}{\stackrel{0}{0}} \underset{\stackrel{0}{0}}{\stackrel{0}{0}}$ & $\underset{-}{\stackrel{0}{8}}$ & 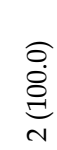 & $\begin{array}{l}\stackrel{0}{0} \\
\stackrel{0}{0} \\
0\end{array}$ \\
\hline
\end{tabular}

